\title{
Orthogonal Matching Pursuit for Enhanced Recovery of Sparse Geological Structures with the Ensemble Kalman Filter
}

\author{
Furrukh Sana $^{a, 1}$, K. Katterbauer ${ }^{b}$, Tariq Al-Naffouri ${ }^{a}$, I. Hoteit ${ }^{a, b}$ \\ ${ }^{a}$ Dept. of Computer, Electrical and Mathematical Sciences and Engineering, \\ ${ }^{b}$ Dept. of Physical Sciences and Engineering, \\ King Abdullah University of Science \&3 Technology, 23955-9600 Thuwal, Saudi Arabia
}

\begin{abstract}
Estimating the locations and the structures of subsurface channels holds significant importance for forecasting the subsurface flow and reservoir productivity. These channels exhibit high permeability and are easily contrasted from the low permeability rock formations in their surroundings. This enables formulating the flow channels estimation problem as a sparse field recovery problem. The Ensemble Kalman filter (EnKF) is a widely used technique for the estimation and calibration of subsurface reservoir model parameters, such as permeability. However, the conventional EnKF framework does not provide an efficient mechanism to incorporate prior information on the wide varieties of subsurface geological structures, and often fails to recover and preserve flow channel structures. Recent works in the area of Compressed Sensing (CS) have shown that estimating in a sparse domain, using algo-
\end{abstract}

\footnotetext{
${ }^{1}$ Email: furrukh.sana@kaust.edu.sa
} 
rithms such as the Orthogonal Matching Pursuit (OMP), may significantly improve the estimation quality when dealing with such problems. We propose two new, and computationally efficient, algorithms combining OMP with the EnKF to improve the estimation and recovery of the subsurface geological channels. Numerical experiments suggest that the proposed algorithms provide efficient mechanisms to incorporate and preserve structural information in the EnKF and result in significant improvements in recovering flow channel structures.

Keywords: Subsurface Characterization, Ensemble Kalman Filter, Compressed Sensing, Orthogonal Matching Pursuit, K-SVD, State-Parameter Estimation, Sparsity. 


\section{Introduction}

Subsurface reservoir characterization is one of the challenging problems encountered in geosciences and is of major interest for petroleum and hydrological applications [1]. This includes the identification of hydrocarbon, geothermal reservoirs, and geological features such as flow channels and fractures [2]. Geological formations are formed by complex processes within the earth, leading to rock channels that exhibit desirable hydraulic properties as compared to the surrounding formations [3].

Rock channel structures separate the rock formation into two distinctive groups, consisting of high permeability flow channels and the surrounding

rock formation whose permeability is orders of magnitude lower [4]. Estimating the locations and the structures of these channels holds significant importance for forecasting the subsurface flow. These high permeability channels serve as preferred pathways for fluid propagation and aquifers may be conveniently placed on top of these channels to optimize reservoir productivity.

Accurate reconstruction of the subsurface geological features is challenging because of the limited number of measurements available from the wells [5]. As a consequence, the estimated features and parameters may significantly differ from the actual representation because of the multiple nonunique solutions reproducing the available data, meaning large uncertainties in the predicted flow and displacement patterns [6]. This has led to the investigation of advanced estimation techniques for enhanced recovery of subsurface reservoir parameters $[4,7,8,9,10]$. 
For the general reservoirs and the spatial scales under consideration, the fraction of the reservoir occupied by high permeability channels is rather limited. The subsurface field mostly comprises of smooth (or piecewise smooth) features exhibiting spatial continuity and long range correlations $[7,11,12,13]$. For features exhibiting such characteristics, sparse transform domains have proven useful for a variety of image reconstruction problems, enabling efficient representation based on a low number of transform domain coefficients $[14,15]$. Careful selection of an appropriate sparse domain for the problem of recovering subsurface geological structures may further help incorporating and preserving structural information in the estimation process and also in reducing the computational complexity of the problem $[4,16,17,13]$.

The Ensemble Kalman filter (EnKF) is a popular Monte Carlo variant of the standard Kalman filter (KF) [18] designed for nonlinear estimation problems. The EnKF is widely used for the estimation of both dynamic (e.g. saturation) and static (e.g. permeability) subsurface reservoir parameters $[19,20,21,22]$. As the KF, the EnKF estimation process is also based on forecast and analysis cycles but differs in the way the first two moments of the system state are represented by an ensemble of state vectors. This Monte Carlo formulation allows to significantly reduce the computational complexity and enables the implementation of the filter with large scale nonlinear systems [18]. However, the conventional EnKF does not provide a computationally efficient mechanism to incorporate prior information on the large 
number of possible subsurface geological features, and often fails to recover and preserve flow channel structures [19].

The conventional EnKF relies mainly on the initial ensemble to enforce any information on the prior model [19]. The ability of the EnKF to recover channel structures by careful selection of the initial ensemble was investigated in [19], concluding that a large ensemble, of 300 or more members, has to be used to obtain adequate reconstruction. The need for a large ensemble set is, however, a severely limiting factor considering the significant computational resources required for integrating each ensemble member forward with the reservoir simulator [23].

Compressive sensing (CS) is changing the manner of treating digital signals, providing efficient tools to improve the reconstruction ability of signals and fields exhibiting sparsity in some domain $[24,25,26]$. CS principals allow for enhanced recovery of sparse domain signals from a reduced number of observations by exploiting the prior information on signal sparsity $[27,25]$. Witnessing tremendous growth in sparse image reconstruction, CS has gained foothold in subsurface estimation [4, 7, 28]. Jafarpour et. al. investigated the use of the well known Discrete Cosine Transform (DCT) in [6] and the Wavelet transforms in [29] for sparse representation within an EnKF framework and showed that such representations may help achieving performances comparable to a spatial domain EnKF while using a much less number of coefficients in the transform domain. However, the gains from representation in these sparse domains are largely limited to the reduction 
in computational complexity and improving the ill-posedness of the problem. A DCT or Wavelet representation does not provide an efficient mechanism for incorporating prior information on the possible structures of the subsurface geological field and their inter-connectivity [13]. Khaninezhad et. al. proposed a hybrid parametrization approach in [17] using generic (DCT) basis functions alongside Principal Component Analysis (PCA) based geologic basis functions. However, as noted by the authors, the PCA basis functions are only suitable for jointly Gaussian random fields. Neither the generic, nor the PCA basis functions, provide an effective parametrization for the more complex geological structures, such as meandering flow channels. Moreover, using a large number of hybrid basis functions can lead to redundancies in representation.

In $[16,30]$, a comprehensive study was presented on the influence of the sparse geologic dictionaries learned over a large set of training images using the K-SVD algorithm. The basis elements from this dictionary define the sparse transform domain for representing the ensemble members and are used to incorporate prior structural information within an inversion process. The main conclusion of these studies was that diverse geologic dictionaries lead to robust estimation attributed to the connectivity exhibited by the dictionary elements. Khaninezhad et. al. in [16] also present a technique to solve the problem based on a variant of the Iterative Recursive LeastSquares (IRLS) algorithm. However, the proposed iterative framework in $[16]$, as well as those in $[7,17,13]$, may require implementing the adjoint 
model for efficient computation of the gradients of the objective function, especially when dealing with real large scale reservoir problems. Coding the adjoint is a challenging and demanding process [31, 32].

Greedy algorithms, such as Orthogonal Matching Pursuit (OMP), offer an alternative to the $\ell_{1}$-norm minimization based CS techniques [33]. Given a dictionary of basis elements representing some sparse domain, OMP attempts to represent the unknown signal using a sparse linear combination of the dictionary elements [34]. The coefficients (or weights) of this linear combination represent the unknown signal in the sparse domain. We propose two new schemes employing the OMP algorithm to enhance the estimation and recovery of the subsurface geological features with the EnKF. We perform various numerical experiments to demonstrate the efficiency of the proposed schemes using a two-phase subsurface flow model. We also conduct sensitivity experiments to study the robustness of the proposed techniques with respect to the ensemble size, observational errors, and number of observations. The main contributions of this work can be summarized as follows:

- We develop two novel methods to efficiently incorporate and preserve structural information in the EnKF estimation process, both resulting in enhanced recovery of the subsurface geological structures.

- We propose the Dynamic Ensemble Update (DEU)-EnKF algorithm which utilizes OMP to enhance the EnKF performance when implemented with small ensembles. The DEU-EnKF algorithm dynamically 
updates and improves the filter ensemble after each filtering cycle by finding members most statistically correlated with the EnKF estimate from a large pool of subsurface geological structures. In contrast with the previous work reported in [19], the proposed scheme is shown here to achieve good performances using much less ensemble members, greatly reducing the computational complexity of the problem.

- We propose the Sparse Geological Structures Domain (SGSD)-EnKF algorithm to exploit sparse geologic dictionaries for the EnKF based subsurface field reconstruction problem. The SGSD-EnKF algorithm transforms the EnKF estimation of the subsurface channel structures to a specifically constructed domain based on sparse geological dictionaries. This domain is learned over a large collection of subsurface geological structures using the K-SVD algorithm, which allows to incorporate useful prior information into the estimation process.

- The two proposed methods are shown to exhibit faster convergence towards the true state of the reservoir, reducing the number of update cycles required to obtain a good reconstruction of the unknown field. They also result in significant reduction in the computational complexity, through compact representation in transform domain for the SGSD-EnKF scheme, and by relaxing the requirement for large ensembles using the DEU-EnKF scheme.

The paper is organized as follows. Section 2 provides a brief overview 
of the simulation setup coupling a two-phase subsurface flow model with an EnKF. Section 3 describes the components of the sparse parametrization framework. The proposed schemes are introduced in Section 4. Section 5 describes the experimental setup and presents and analyzes the simulations results. The paper concludes with a general discussion and summary of the main results.

\section{Subsurface Characterization and EnKF}

This section presents the subsurface reservoir parameter estimation problem based on the MATLAB Reservoir Simulation Toolbox (MRST) and the EnKF as outlined in Figure 3.

\subsection{Reservoir Simulator}

Amongst the most common reservoirs are water driven reservoirs whose pressure is above the bubble point pressure, and hence only a water and an oil phase is present in the reservoir. Assuming that the oil does not exhibit strongly different properties for different pressure levels, a two-phase model approach is generally accepted and is used in this paper.

MRST is a 2D finite difference two-phase flow reservoir simulator [35] coupling a well model to the two-phase flow problem for the oil and water phases governed by the system of equations [36]

$$
\nabla \cdot v=q
$$




$$
v=-K\left[\lambda \nabla p+\left(\lambda_{w} \rho_{w}+\lambda_{o} \rho_{o}\right) g \nabla z\right]
$$

and

$$
\phi \frac{\partial s_{w}}{\partial t}+\nabla \cdot\left(f_{w}\left(s_{w}\right)\left[v+\lambda_{o}\left(\rho_{o}-\rho_{w}\right) g K \nabla z\right]\right)=q_{w}
$$

where $\lambda$ is the mobility of the fluid with $\lambda_{o}$ and $\lambda_{w}$ denoting the individual mobilities for the oil and water phase $(c P)^{-1}, \rho_{o}, \rho_{w}$ the densities $\left(k g / m^{3}\right), f_{w}$ the fractional flow of the water phase (dimensionless), and $s_{w}, s_{o}$ the water and oil saturation (dimensionless) respectively, with $s_{o}+s_{w}=1$. Furthermore, $q$ represents the flux $\left(\mathrm{kg} / \mathrm{m}^{3} / \mathrm{s}\right), \phi$ porosity of the formations (dimensionless), $v$ Darcy's velocity $(\mathrm{m} / \mathrm{s}), \mathrm{g}$ the gravity $\left(\mathrm{m} / \mathrm{s}^{2}\right), K$ the permeability tensor $\left(m^{2}\right)$, and $p$ the pressure within the reservoir (bars). The system is solved sequentially, first computing the solution of Equation (1) and (2) using fixed saturation values for fluxes and pressure, and then evolves saturation values with the computed fluxes and pressure levels according to Equation (3). This reservoir simulator acts as the forward model, integrating each ensemble member of the EnKF forward in time.

Modern reservoir simulators incorporate a variety of different parameters, such as fluid properties, rock formation types, fault parameters and well locations. While fluid properties may be determined in lab studies through samples, and the properties of the wells may be determined from well logs and tests, determining rock formation properties and structures is more difficult and typically need to be estimated. 


\subsection{Ensemble Kalman Filter (EnKF)}

The sparse subsurface reservoir history matching framework interfaces the MRST simulator [35] together with a compressed sensing estimation proce-

dure based on the EnKF template provided by O. Leeuwenburgh [37]. The problem can be formulated using the state-space model

$$
\begin{gathered}
\mathbf{x}_{t+1}=\mathbf{M}_{t}\left(\mathbf{x}_{t}\right)+\boldsymbol{\eta}_{t}, \\
\mathbf{y}_{t}=\mathbf{h}_{t}\left(\mathbf{x}_{t}\right)+\boldsymbol{\epsilon}_{t},
\end{gathered}
$$

where $\mathbf{M}_{t}$ represents the reservoir simulator with the state vector $\mathbf{x}_{t}=$ $\left[\mathbf{k}_{t}, \boldsymbol{\phi}_{t}, \mathbf{p}_{t}, \mathbf{s}_{t}\right]$ consisting of vectors of the static parameters, permeability $\mathbf{k}$ and porosity $\boldsymbol{\phi}$, and dynamic variables, pressure $\mathbf{p}$ and saturation $\mathbf{s}$ defined over the entire grid space at time $t$, and $\boldsymbol{\eta}_{t}$ a stochastic term representing the model error. $\mathbf{y}_{t}$ is the observation that is related to the state via the (nonlinear) observation operator $\mathbf{h}_{t}$, perturbed by a random noise $\boldsymbol{\epsilon}_{t}$. The observation is composed of the production data, including the flux, the bore hole pressure (bhp), and the water cut ratio (wct), acquired using their respective sensors at all well locations. Both $\boldsymbol{\eta}_{t}$ and $\boldsymbol{\epsilon}_{t}$ are assumed independent and normally distributed of mean zero and covariance matrices $\mathbf{Q}_{t}$ and $\mathbf{R}_{t}$, respectively.

First introduced by Evensen et. al. [38], the EnKF has been ever since extensively applied in the field of reservoir history matching [20, 39, 40, 41]. Being fundamentally based on the Kalman Filter (KF), the EnKF differs 
from the $\mathrm{KF}$ in that the distribution of the system state is represented by a set, or ensemble, of state vectors approximating the KF estimate and its error covariance matrix by the ensemble sample mean and covariance. Despite the fact that the EnKF updates are only based on means and covariances and that these covariances are generally computed from finite size ensembles, the EnKF was shown to perform well for a variety of history matching problems $[20]$.

In order to achieve efficient computation and to handle nonlinear observations, we have employed an observation matrix-free implementation of the EnKF. Let $N_{e}$ be the ensemble size and $\mathbf{X}_{t}=\left[\mathbf{x}_{1, t}, \ldots, \mathbf{x}_{N_{e}, t}\right]$ the state ensemble matrix at time step $t$, with $\mathbf{x}_{i, t}$ denoting the state vector of the $i$-th ensemble member. Further, define the covariance anomaly $\mathbf{A}_{t}=$ $\mathbf{X}_{t}-\frac{1}{N_{e}}\left(\sum_{i=1}^{N_{e}} \mathbf{x}_{i, t}\right) \mathbf{e}_{1 \times N_{e}}$ with $\mathbf{e}_{1 \times N_{e}}$ denoting the matrix with ones as elements and size $1 \times N_{e}$, and $\left[\mathbf{H}_{t}\right]_{:, i}=\mathbf{h}_{t}\left(\mathbf{x}_{i, t}\right)-\frac{1}{N_{e}} \sum_{j=1}^{N_{e}} \mathbf{h}_{t}\left(\mathbf{x}_{j, t}\right)$ the observation matrix with $\mathbf{h}_{t}\left(\mathbf{x}_{i, t}\right)$ being the observation prediction for the $i$-th ensemble state vector. Then, for the data matrix $\mathbf{D}_{t}$ whose columns are the observations perturbed with noise sampled from the distribution of $\boldsymbol{\epsilon}_{t}$, and the corresponding ensemble covariance matrix $\tilde{\mathbf{R}}_{t}$, the EnKF update step uses the observation to compute the analysis ensemble $\mathbf{X}_{t}^{a}$ from the forecast ensemble $\mathbf{X}_{t}^{f}$ as

$$
\mathbf{X}_{t}^{a}=\mathbf{X}_{t}^{f}+\frac{1}{N_{e}-1} \mathbf{A}_{t} \mathbf{H}_{t}^{T}\left(\frac{1}{N_{e}-1} \mathbf{H}_{t} \mathbf{H}_{t}^{T}+\tilde{\mathbf{R}}_{t}\right)^{-1}\left(\mathbf{D}_{t}-\mathbf{h}_{t}\left(\mathbf{X}_{t}^{f}\right)\right)
$$


The forecast ensemble members are then obtained by integrating the most recent analysis ensemble members forward in time with the reservoir simulator using (4) before an update step is applied with the new incoming observation. For further details about the EnKF, the reader may refer to the review articles of Aanonsen et. al. [20], Luo and Hoteit [42], and Hoteit et. al. [43].

\section{Sparse Domain Representation}

Compressed Sensing (CS) methods have been shown to significantly improve the estimation quality of signals exhibiting sparsity in some domain $[24,25,44]$. CS approaches rely either on $\ell_{1}$-norm minimization methods [7], or on greedy pursuit algorithms [4] to compute the sparse solution. $\ell_{1^{-}}$ norm minimization based techniques try to reduce the residual error while constraining the $\ell_{1}$-norm of the solution vector. Greedy algorithms attempt to achieve the same using heuristic approaches while limiting the maximum number of non-zero values in the solution vector.

For large scale nonlinear reservoir problems, methods relying on $\ell_{1}$-norm minimization approaches requires the implementation of the adjoint model in order to efficiently compute the gradients of the objective function $[7,17,13]$, which is a very challenging and demanding process [31, 32]. Orthogonal Matching Pursuit (OMP) is a greedy algorithm that is easy to implement and was shown to provide an efficient method to compute the solution to a sparse problem [33]. We first describe the OMP algorithm explaining its 
mechanism of finding a sparse solution given a specified dictionary. We then present the K-SVD dictionary learning algorithm to generate a sparse basis from a large set of subsurface geological structures.

\subsection{OMP Algorithm}

Given an estimate of sparsity $p$ for a signal (or field) represented by a vector of unknown weights $\mathbf{x}$ in a $d$-dimensional space, OMP attempts to reconstruct the data signal $\mathbf{y}$ using a linear combination of at most $p$ basis elements (atoms) from an over-complete dictionary $\boldsymbol{\Psi}$ [33]. The dictionary is a collection of basis elements representing a particular domain for signal representation and can be chosen to suit a particular reconstruction problem [45]. OMP is easy to implement and can provide effective computations of the sparse solution. This has led to its application to numerous sparse signal reconstruction problems $[4,46,34]$.

The OMP algorithm solves the minimization problem

$$
\min _{\mathbf{x}}\|\mathbf{x}\|_{\mathbf{0}} \quad \text { subject to } \quad\left\|\mathbf{y}-\Psi_{\mathbf{x}}\right\|_{2}<\eta
$$

by iteratively projecting the data $\mathbf{y}$ onto a dictionary $\boldsymbol{\Psi}$ and finding the basis elements that are most correlated with the residuals. The term $\|\mathbf{x}\|_{\mathbf{0}}$ denotes the $\ell_{0}-$ norm and represents the sparsity level of $\mathbf{x}$, i.e. the number of its non-zero elements, and $\eta$ is the error tolerance. The OMP algorithm is outlined in Algorithm 1. For further information on the OMP algorithm, the reader may refer to [33]. 


Algorithm 1 Orthogonal Matching Pursuit (OMP) Algorithm

- Input: Data vector $\mathbf{y}$, dictionary $\boldsymbol{\Psi}$, sparsity estimate $p$, error threshold $\eta$

- Initialize: Set $t=0, \mathbf{x}_{\mathbf{0}}=\mathbf{0}$, index set $\boldsymbol{\Delta}_{\mathbf{0}}=\mathbf{0}$, and residual $\mathbf{r}_{\mathbf{0}}=\mathbf{y}$

- While $t<p$ or $\left\|\mathbf{r}_{t-1}\right\|_{2}<\eta$

- Find the index of the basis element most correlated with the residual

$$
\delta_{t}=\underset{j=1, \ldots, d}{\arg \max }\left|\left\langle\mathbf{r}_{t-1}, \psi_{j}\right\rangle\right|
$$

- Augment the index set

$$
\boldsymbol{\Delta}_{t}=\boldsymbol{\Delta}_{t-1} \cup \delta_{t}
$$

- Solve the least-squares problem

$$
\mathbf{x}_{t}=\underset{\mathbf{x}}{\arg \min }\left\|\mathbf{y}-\mathbf{\Psi}_{\mathbf{\Delta}_{t}} \mathbf{x}\right\|_{2}
$$

- Calculate new residual

$$
\mathbf{r}_{t}=\mathbf{y}-\Psi_{\Delta_{t}} \mathbf{x}_{t}
$$

- End While

- Output: Final solution $\mathbf{x}_{t}$, index set $\boldsymbol{\Delta}_{t}$, and residual $\mathbf{r}_{t}$

\subsection{Dictionary Learning}

A key factor in sparse estimation techniques is the identification of a domain (basis) in which the signal, or field, of interest can be modeled as sparse. Sparse domain means that any signal in that domain could be represented by a linear combination of a few basis elements only [25]. Discrete Cosine Transform (DCT) [47] and Wavelets [48] are two of such sparse domains. However, 
a sparse domain can also be constructed to suit a particular application, and algorithms used for such purpose are called dictionary learning algorithms [49]. Given a set of training signals (or fields) $\mathbf{Y}$ of size $N_{t}$, dictionary learning algorithms attempt to find the basis elements to construct a dictionary $\Psi$ whose sparse linear combination can represent each of the signals (or fields) in the training set $\mathbf{Y}$. The weights of this sparse linear combination, for each signal in the training set, are then given by the corresponding vector of coefficients in a set $\mathbf{X}$. Mathematically, dictionary learning algorithms attempt to solve the optimization problem [45]

$$
\min _{\mathbf{X}, \mathbf{\Psi}}\left\{\|\mathbf{Y}-\mathbf{\Psi} \mathbf{X}\|_{2}^{2}\right\} \text { s.t. }\left\|\mathbf{x}_{i}\right\|_{0} \leq \mathbf{T}_{0} \forall i=1, \ldots, N_{t}
$$

where $\mathbf{T}_{0}$ represents the desired number of nonzero entries for each of the signals in $\mathbf{X}$. In contrast to OMP, the optimization problem above attempts to minimize the representation error for all signals in the training set $\mathbf{Y}$ by not only finding the weights $\mathbf{X}$ of the basis elements in $\boldsymbol{\Psi}$, but also by adjusting the basis elements themselves. This is a non-convex problem and NP-hard to solve [4].

K-SVD is a dictionary learning algorithm that solves the problem defined in equation (8) using a recursive two-step approach [45]. In the first step, given an initial dictionary $\Psi^{j-1}$, where the superscript $j$ denotes the iteration count, the algorithm tries to find the weights $\mathbf{X}^{j}$ that best approximate each of the signals in the training set $\mathbf{Y}$. This step can be solved using either a 
$\ell_{1}-$ norm minimization technique or a greedy algorithm such as OMP. In the second step, K-SVD updates the dictionary $\mathbf{\Psi}^{j}$ while keeping the weights $\mathbf{X}^{j}$ fixed. The process iterates until a given representation error tolerance for all signals in the training set, or the maximum number for iterations is reached. For further details about the K-SVD algorithm, the reader may refer to [45].

\section{Methodology}

This section presents our two proposed schemes combining OMP and the EnKF for enhanced estimation of subsurface channels.

\subsection{Dynamic Ensemble Update EnKF (DEU-EnKF)}

The performance of the EnKF strongly depends on the prior ensemble [18]. As reported in [19], the ensemble members should be chosen such that they closely resemble the unknown field to be estimated. In reality, however, the true structure of the field cannot be known a priori and the generated initial ensemble members may be far from the true field. [19] further concluded that a large ensemble of 300 or more members should be used in order to achieve adequate reconstruction of the reservoir channels. However, using such a large ensemble is computationally expensive since each ensemble member needs to be integrated with the reservoir simulator [50]. Additionally, given the limited number of well data, the EnKF update may not be efficient enough for fast convergence towards the true solution. One may consider to dynamically update the ensemble members during the 
EnKF assimilation cycles, such that the ensemble members become more closely related to the unknown field over subsequent filtering cycles. The OMP algorithm provides an efficient mechanism to achieve this task.

Given a dictionary $\Psi$ whose atoms represent realizations of the subsurface geological structures, and an estimate of the unknown permeability field $\mathbf{k}$, one could use the OMP algorithm to select the atoms of the dictionary that most closely resemble the unknown field. These atoms then serve as the updated set of ensemble members $\mathbf{K}$ for the permeability field in the next filtering cycle. We use the mean EnKF estimate of the permeability field $\mathbf{k}_{t}$ at time $t$ as the data $\mathbf{y}$ in Algorithm 1 and use the OMP algorithm to find the basis elements of the dictionary $\Psi$ most statistically correlated with that estimate. The sparsity parameter $p$ in the OMP algorithm is set by the ensemble size, i.e. $p=N_{e}$. By iteratively projecting the residual onto the dictionary $\Psi, \mathrm{OMP}$ will select the basis elements most correlated with the EnKF estimate from a large pool of realizations of the subsurface geological structures. The basis elements are represented by the index set $\boldsymbol{\Delta}$ in Algorithm 1 and serve as the new set of ensemble members $\mathbf{K}_{t+1}$ for the next filtering cycle at time $t+1$. The ensemble is enhanced after each filtering cycle, replacing the members by new elements from $\Psi$ based on their similarity with the EnKF estimate of the permeability field. The proposed algorithm enables the use of a small ensemble size while accounting for a large number of possible subsurface geological structures of the unknown field, thereby significantly reducing the computational requirements. This 
should also speed up the convergence of the EnKF solution and enable greater ability of overcoming the effects of inaccurate initialization. The proposed algorithm is outlined in Algorithm 2.

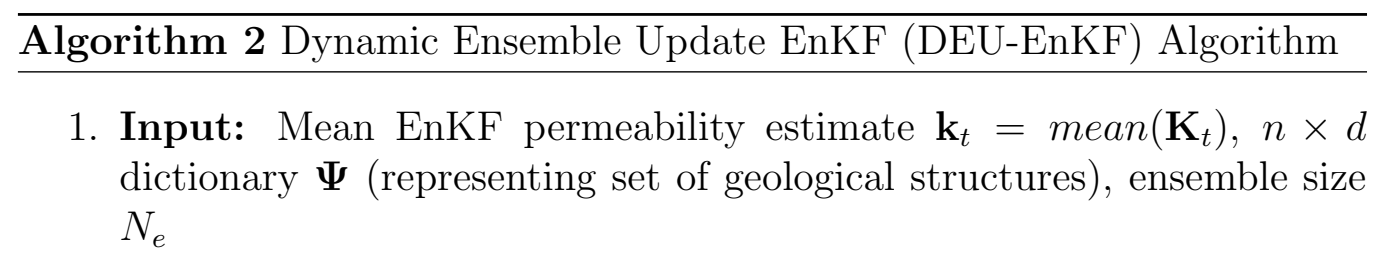

2. Set data $\mathbf{y}_{t}=\mathbf{k}_{t}$ and sparsity level $p=N_{e}$

3. Find the index set for the new permeability ensemble

$$
\left[\boldsymbol{\Delta}_{t+1}\right]=\mathbf{O M P}\left(\mathbf{y}_{t}, \Psi, p\right)
$$

4. Retrieve new members from the dictionary

$$
\mathbf{K}_{t+1}=\Psi_{\Delta_{\mathbf{t}+\mathbf{1}}}
$$

\section{Output: $\mathbf{K}_{t+1}$}

\subsection{Sparse Geological Structures Domain EnKF (SGSD-EnKF)}

By transforming the ensemble members to a sparse domain, the benefits of sparse domain representation can be exploited by the EnKF. An efficient and robust sparse basis for representing the permeability ensemble can be constructed based on the K-SVD algorithm presented in Section 3.2 to incorporate useful prior information about a large number of different possible subsurface geological structures into the estimation process.

A large set of realizations, each depicting a different subsurface geological 
field structure, can be used to train the K-SVD algorithm to generate the sparse basis represented by dictionary $\boldsymbol{\Psi}$. The OMP algorithm of Section 3.1 can then be applied to transform the permeability ensemble $\mathbf{K}_{t}^{f}$ in the spatial domain into the new sparse domain. The coefficients (or weights) used for the sparse linear combination in the OMP algorithm represent the permeability in the new sparse domain. This sparse domain permeability $\boldsymbol{\kappa}_{t}$ is then augmented with the ensembles for other state variables, forming the new state vector

$$
\mathbf{X}_{t}=:\left[\boldsymbol{\kappa}_{t}, \boldsymbol{\phi}_{t}, \mathbf{P}_{t}, \mathbf{S}_{t}\right] \text {, }
$$

which is then used in the EnKF filtering process.

The channelized structures and the connectivity exhibited by the basis elements in the new sparse domain make it a natural choice for sparse representation of the permeability ensemble. The permeability ensemble is transformed back to the spatial domain after each EnKF update step for integrating it forward in the next forecast step. It is noted that since permeability is a static model parameter (not a dynamic state variable) and remains unchanged during the forecast step, the sparse coding step for the permeability ensemble is required just once, at the first filtering cycle. The proposed algorithm, referred to as the Sparse Geological Structures Domain EnKF (SGSD-EnKF), is summarized in Algorithm 3. 
$\overline{\text { Algorithm } 3 \text { Sparse Geological Structures Domain EnKF (SGSD-EnKF) }}$ Algorithm

- Input: Forward permeability ensemble $\mathbf{K}_{t}^{f}$, dictionary $\boldsymbol{\Psi}$ (generated once using K-SVD), observation data $\mathbf{y}_{t}$, sparsity level $p$.

- Transform to sparse domain

- If $t=1$

$$
\boldsymbol{\kappa}_{t}^{f}=\operatorname{OMP}\left(\mathbf{K}_{t}^{f}, \boldsymbol{\Psi}, p\right)
$$

- else

$$
\boldsymbol{\kappa}_{t}^{f}=\boldsymbol{\kappa}_{t-1}^{a}
$$

- Construct the new state vector

$$
\mathbf{X}_{t}^{f}=:\left[\boldsymbol{\kappa}_{t}^{f}, \boldsymbol{\phi}_{t}^{f}, \mathbf{P}_{t}^{f}, \mathbf{S}_{t}^{f}\right]
$$

- Perform EnKF analysis with observation data $\mathbf{y}_{t}$

$$
\mathbf{X}_{t}^{a}=\operatorname{EnKF}\left(\mathbf{X}_{t}^{f}, \mathbf{y}_{t}\right)
$$

where

$$
\mathbf{X}_{t}^{a}=:\left[\boldsymbol{\kappa}_{t}^{a}, \boldsymbol{\phi}_{t}^{a}, \mathbf{P}_{t}^{a}, \mathbf{S}_{t}^{a}\right]
$$

- Transform back to spatial domain

$$
\mathbf{K}_{t}^{a}=\mathbf{\Psi} * \boldsymbol{\kappa}_{t}^{a}
$$

- Output: $\mathbf{K}_{t}^{a}$

\section{Experimental Setup and Simulation Results}

\subsection{Setup}

The reference reservoir permeability field is shown in Figure 1-(a) where the reservoir is spread $0.5 \mathrm{~km}$ in both $\mathrm{x}$ and $\mathrm{y}$-directions and $10 \mathrm{~m}$ in the $\mathrm{z}$ - 
direction. The physical domain has been divided into 50 cells in both $\mathrm{x}$ and y directions and a single cell in z-direction. The reference reservoir consists of flow channels and rock formations. The channels through which the oil flows exhibit high permeability values of 5000 millidarcy and are indicated by the red color. The rock features surrounding the flow channels on the other hand exhibit low permeability values of 100 millidarcy and are indicated by the blue color.

Figure 1 also shows the injector-producer patterns used in the history matching experiments. The term injector-producer pattern refers to the way the injector and producer wells are distributed over the reservoir domain. The injector wells are used to pump down water to the subsurface reservoir and the producer wells are then used to pump out oil from the reservoir. Different injector-producer patterns may result in different production levels and estimation performances. To evaluate the performance of our proposed algorithms and demonstrate their robustness, we have tested three different injector-producer patterns as shown in the panels (b),(c), and (d) of Figure 1, where the black circles indicate an injector well and a white circle indicates a producer well. All experiments were performed on a Intel Xeon X5550 2.67 GHz machine using 6 parallel CPUs.

Figure 2 summarizes the steps for generating multiple realizations of subsurface geological structures followed by a dictionary learning process based on these realizations. The initial input is a training image (shown in Figure 2(a)) that is used to generate 2000 realizations of subsurface geological 
structures (partially shown in Figure 2(b)) using the SNESIM (Single Normal Equation SIMulation) algorithm [51]. The main aspect of this algorithm is that the probability model for generating realizations is determined from a training image, hence preserving the channel structures and connections. The training image used has a resolution of $239 \times 239$ with the individual realizations being of size $50 \times 50$. The initial ensemble for the filters was randomly selected from this large set of realizations. These realizations are further used in our proposed algorithms in two ways: (i) for subsequent updates of the ensemble members in the proposed DEU-EnKF algorithm, and (ii) as an input training set for the K-SVD algorithm to generate the dictionary of 1000 linearly independent basis elements (partially shown in Figure 2(c)) for the SGSD-EnKF scheme. The size of the state vector in the new SGSD domain is hence reduced to $N=1000$ compared to $N=2500$ in the spatial domain. Finally, this dictionary becomes the input for the OMP algorithm, which provides a weighted linear combination of the dictionary basis elements (depicted in Figure $2(\mathrm{~d})$ ) to re-generate any of the original realizations, or represent them in the K-SVD dictionary domain.

The wells configuration depicted by the injector-producer pattern $\mathrm{A}$ in Figure 1 has been used in the majority of the experiments and possesses one injector well (black circle) at the center of the domain and four producing wells (white circles) at the corners. Reference flow and production fields were generated with the reservoir simulator based on the reference permeability field shown in Figure 1. Porosity values were assumed to be known and 
constant at 30\% value, and hence not included in the estimation process. Bottom hole pressure (BHP), water cut ratio (WCR), and production flux data are extracted from the reference fields and perturbed with independent noise and then used as observations. The observation error covariance matrix $\mathbf{R}_{k}$ is hence diagonal. Observational error standard deviation was set to 290 psi for BHP, $25 \mathrm{~m}^{3} /$ day for the flux and around $7 \%$ measurement for WCR, inline with realistic data. Production data are collected every 30 days over a total history matching period of 5 years. The initial pressure levels within the reservoir were set at 5076 psi to maintain a pressure level of $4350 \mathrm{psi}$ in the producing wells. The model is assumed perfect, i.e. $\mathbf{Q}_{k}=0$. This setup was adopted in a series of history matching experiments for estimating the permeability field and recovering the channel structures based on the production data. This history matching process is illustrated in Figure 3.

In order to assess the accuracy of the recovered channel structures and locations, we have used two different criterions for evaluating the channels recovery by the different filtering schemes. We define the Channel Estimation Error (CEE) as

$$
C E E=\frac{\sum_{i=1}^{N}\left|y_{i}^{r e f}-y_{i}^{e s t}\right|}{N},
$$

where $y_{i}^{b}(b=r e f$,est $)$ is a binary variable indicating whether the $i$-th estimated non-zero location is part of the channels or not. A threshold value is used to generate binary versions of the reference and estimated permeability 
fields in order to perform CEE calculation. The CEE measures the recovered field matching of the reference permeability field in terms of its non-zero support. A low value of CEE is indicative of low estimation error, and therefore good recovery.

We also adopt a technique from the image processing community to compare the structural information of two images. The structural similarity (SSIM) index [52] is motivated by the human visual system and has been shown to correlate very well with the opinion scores from human observers [53]. It performs a pixel-wise comparison of the luminance, contrast and structural information in two images. The structural comparison is based on the measure of correlation, as given by the inner product, between the two normalized images. A matlab code for calculating the SSIM index is available online and is used here for the structural comparison of the estimated fields with the reference field. A high SSIM value indicates more similarity between the two fields and hence good quality of estimation. More information on this technique can be found in [52].

\subsection{Subsurface Flow Model Parameter Estimation}

Figure 4 presents the yearly estimates of the permeability fields over the 5years history matching period using the standard EnKF and the two proposed sparse EnKF schemes, DEU-EnKF and SGSD-EnKF. The injector-producer pattern A shown in Figure 1 is used here and all filters were implemented with an ensemble size of 80 members. As evident from the images, there is a clear 
mismatch in the channels estimated by the standard EnKF where only small traces of the channels are recovered at the bottom of the domain, but not at the correct locations. The performance of the two proposed techniques is much better, progressively improving over the history matching period. This is also confirmed by their respective CEE and SSIM values plotted in Figure 5. The recovered fields exhibit similar structures to the reference permeability field and the flow channels in Figure 1 while using a relatively small ensemble of just 80 members.

The additional one-time step of transforming the ensemble set to the SGSD domain adds only about 2 minutes to the overall simulation time of 5.5 hours for an ensemble of 80 members and a sparsity rate of $10 \%$. A sensitivity analysis for the SGSD-EnKF scheme against other sparsity rates, in terms of the SSIM index, is shown in Figure 6. Sparsity rates higher or lower than $10 \%$ resulted in degraded reconstruction, a consequence of using too little or too many basis elements. For the DEU-EnKF scheme, the sparsity parameter for the OMP algorithm sets the desired number of ensemble members to choose from the dictionary, i.e. the ensemble size. The DEU-EnKF scheme only added about 53 seconds in total to the entire simulation time for the case of finding 80 new ensemble members. A threshold value of 3500 millidarcy was used to generate the binary versions of the estimated fields for calculating the CEE. A sensitivity analysis for the CEE values at the end of the 5-years history matching period against different threshold values is given in Figure 7 and shows that the proposed schemes outperform the standard EnKF for 
any value of the threshold above 1500 millidarcy.

To evaluate the performance of the SGSD-EnKF against those based on standard sparse domain representations, we also analyze the subsurface channels recovery performance based on the DCT transform representation of the permeability ensemble. A dictionary of DCT basis functions is generated and used instead of the K-SVD based geologic dictionary in the SGSD-EnKF algorithm with a sparsity rate of $10 \%$. Figure 8 shows the permeability fields recovered at the end of 5-years of history matching period with the standard spatial, DCT, and SGSD domain EnKFs, respectively. The figure highlights the limitations of DCT representation, providing no significant enhancement in the reconstruction of the subsurface geological features compared to the standard EnKF. As reported in [6], the DCT representation can help recovering some large-scale features of the permeability field, but also may omit the more small-scale details. This introduces smoothing effects which, although slightly improves the spatial continuity in the estimates, may spread inaccurate channel features and further degrade the overall recovered permeability field. This phenomenon can also be observed in the field estimated based on the DCT representation in Figure 8. Similar results can be expected with Wavelet basis functions as has been reported in [29].

Figure 9 outlines the performance of the three techniques against different ensemble sizes. The quantitative analysis, in terms of CEE, is presented in Figure 10. As expected, the performance of the standard EnKF improves with increased ensemble sizes. However the proposed techniques, particu- 
larly the DEU-EnKF, still exhibit a clear advantage over the standard EnKF for any given ensemble size. The standard EnKF performance is comparable with that of SGSD-EnKF only for certain ensemble sizes. The DEU-EnKF scheme is able to recover significant parts of the channels even when implemented with as low as 40 ensemble members. This highlights the strength of the technique in recovering an unknown field while using a very small ensemble, reducing both the computational complexity and convergence time. The DEU-EnKF provides the best estimate with an ensemble of 40 members and the SGSD-EnKF with an ensemble of 80. The increase in CEE values for larger ensemble sizes could be explained by the enforcement of redundant and unnecessary information arising from the use of more ensemble members. We further analyze the robustness of the standard EnKF and the two proposed techniques against increasing values of the observational error variance as summarized in Table 1. All filters were implemented with 80 ensemble members. The qualitative and quantitative results of these experiments are presented in Figure 11 and Figure 12, respectively. The proposed techniques exhibit robust performances, being weakly sensitive to increased observational errors variance. The performance of the standard EnKF seems to slightly improve in the last case of Table 1, possibly a result of the EnKF relying more on the simulator forecast estimates over noisy observation data. The estimation quality and channel recovery for both proposed techniques is still significantly better than that of the standard EnKF and provides reliable performance while efficiently filtering out observational noise. 
Lastly, we analyze the performance of the filters against the number of observations available for history matching in Figure 13 and 14. We use wells setups based on the injector-producer patterns B and C as shown in Figure 1 to study the effect of using more data on the channel recovery results. History matching more data generally results in significant improvement in the standard EnKF performance. However, quantitative analysis suggests that the standard EnKF performance with injector-producer pattern $\mathrm{C}$ is still not better than that of the SGSD-EnKF scheme even when only history matching the more sparse data from the injector-producer pattern A. This highlights the ability of the proposed scheme to provide good reconstruction of the subsurface field using much less data compared to the standard EnKF. For DEU-EnKF, more data results in even further improved recovery of the channel structures as suggested by the decreasing CEE values. This demonstrates the robustness of the technique in providing significantly enhanced channel recovery with varying number of observations and well configurations.

\section{Summary and Discussion}

We proposed two new methods combining the Orthogonal Matching Pursuit algorithm with the Ensemble Kalman Filter for efficient estimation of channelized subsurface geological structures. The first method enables faster convergence towards the solution by dynamically updating the ensemble set after each filtering cycle and allows recovering the unknown field using small ensemble sizes while taking into account a wide variety of geological struc- 
tures. The second scheme transforms the EnKF estimation of the subsurface channel structures to a specifically constructed domain based on sparse geological dictionaries, enabling problem representation using much less number of variables and incorporation of useful prior information into the estimation process. Our numerical results demonstrate the effectiveness of the two proposed schemes and show clear advantages over the conventional EnKF in recovering the flow channel structures. Both schemes strongly enhance the EnKF performance when dealing with channelized reservoir applications using limited and noisy observations.

Remote sensing techniques, such as InSAR and electromagnetics (EM), are receiving growing interest for reservoir monitoring $[2,54]$ and have also been investigated for reservoir history matching [55] and reservoir characterization [56] purposes. Incorporating such remote sensing data in the proposed sparse recovery frameworks, along with the use of multiple training images to enable recovery of more diverse channel structures, may potentially lead to even further improved recovery of the subsurface channels by enabling effi-

cient exploitation of additional information accounting for the sparse nature and diversity of the subsurface channel structures. This will be investigated in future research.

\section{Acknowledgement}

This work was funded by King Abdullah University of Science and Technology (KAUST), Thuwal, Saudi Arabia. 


\section{References}

[1] D. S. Oliver and Y. Chen, "Recent progress on reservoir history matching: a review," Computational Geosciences, vol. 15, no. 1, pp. 185-221, 2011.

[2] K. Katterbauer, I. Hoteit, and S. Sun, "EMSE: Synergizing EM and seismic data attributes for enhanced forecasts of reservoirs," Journal of Petroleum Science and Engineering, 2014.

[3] M. J. Ronayne, S. M. Gorelick, and J. Caers, "Identifying discrete geologic structures that produce anomalous hydraulic response: An inverse modeling approach," Water Resources Research, vol. 44, no. 8, p. W08426, 2008.

[4] A. Elsheikh, M. Wheeler, and I. Hoteit, "Sparse calibration of subsurface flow models using nonlinear orthogonal matching pursuit and an iterative stochastic ensemble method," Advances in Water Resources, vol. 56, no. 0, pp. 14-26, 2013.

[5] O. M. Bucci, L. Crocco, T. Isernia, and V. Pascazio, "Subsurface inverse scattering problems: quantifying, qualifying, and achieving the available information," Geoscience and Remote Sensing, IEEE Transactions on, vol. 39, no. 11, pp. 2527-2538, Nov. 2001.

[6] B. Jafarpour and D. B. McLaughlin, "History matching with an ensemble Kalman filter and discrete cosine parameterization," Computational Geosciences, vol. 12, no. 2, pp. 227-244, 2008.

[7] B. Jafarpour, V. K. Goyal, D. B. McLaughlin, and W. T. Freeman, "Compressed history matching: exploiting transform-domain sparsity for regularization of nonlinear dynamic data integration problems," Mathematical Geosciences, vol. 42, no. 1, pp. 1-27, 2010.

[8] Y. Yu and L. Carin, "Three-Dimensional Bayesian Inversion With Application to Subsurface Sensing," Geoscience and Remote Sensing, IEEE Transactions on, vol. 45, no. 5, pp. 1258-1270, May 2007.

[9] P. M. Wong, T. D. Gedeon, and I. J. Taggart, "An improved technique in porosity prediction: a neural network approach," Geoscience and 
Remote Sensing, IEEE Transactions on, vol. 33, no. 4, pp. 971-980, Jul. 1995.

[10] B. Wohlberg, D. M. Tartakovsky, and A. Guadagnini, "Subsurface characterization with support vector machines," Geoscience and Remote Sensing, IEEE Transactions on, vol. 44, no. 1, pp. 47-57, Jan. 2006.

[11] L. Li and B. Jafarpour, "Effective solution of nonlinear subsurface flow inverse problems in sparse bases," Inverse Problems, vol. 26, p. 105016, 2010 .

[12] B. Jafarpour, V. K. Goyal, D. B. McLaughlin, and W. T. Freeman, "Transform-domain sparsity regularization for inverse problems in geosciences," Geophysics, vol. 74, no. 5, pp. R69—R83, 2009.

[13] M.-r. Khaninezhad, B. Jafarpour, and S. California, "Sparse Geologic Dictionaries for Field-Scale History Matching Application," no. February, pp. 23-25, 2015.

[14] J.-L. Starck, M. Elad, and D. L. Donoho, "Image decomposition via the combination of sparse representations and a variational approach," Image Processing, IEEE Transactions on, vol. 14, no. 10, pp. 1570-1582, Oct. 2005.

[15] M. B. Wakin, J. K. Romberg, H. Choi, and R. G. Baraniuk, "Waveletdomain approximation and compression of piecewise smooth images," Image Processing, IEEE Transactions on, vol. 15, no. 5, pp. 1071-1087, May 2006.

[16] M. M. Khaninezhad, B. Jafarpour, and L. Li, "Sparse geologic dictionaries for subsurface flow model calibration: Part I. Inversion formulation," Advances in Water Resources, vol. 39, pp. 106-121, 2012.

[17] M. Khaninezhad, B. Jafarpour, and M. M. Khaninezhad, "Hybrid Parameterization for Robust History Matching," SPE Journal, no. June, 2013. [Online]. Available: https://www.onepetro.org/journalpaper/SPE-146934-PA

[18] G. Evensen, "The Ensemble Kalman Filter: Theoretical formulation and practical implementation," Ocean Dynamics, 
vol. 53, no. 4, pp. 343-367, Nov. 2003. [Online]. Available: http://link.springer.com/10.1007/s10236-003-0036-9

[19] B. Jafarpour, D. B. McLaughlin, and Others, "Estimating channelizedreservoir permeabilities with the ensemble Kalman filter: The importance of ensemble design," Spe Journal, vol. 14, no. 02, pp. 374-388, 2009 .

[20] S. Aanonsen, D. Oliver, A. Reynolds, and B. Vallis, "The Ensemble Kalman Filter in Reservoir Engineering-a Review," SPE Journal, vol. 14, no. 3, pp. 393-412, 2009.

[21] A.-A. Lorentzen, R. J., Naevdal, G., Valles, B., Berg, A., \& Grimstad, "Analysis of the Ensemble Kalman Filter for Estimation of Permeability and Porosity in Reservoir Models," Society of Petroleum Engineers., 2005.

[22] L. Duan, C. Farmer, I. Hoteit, X. Luo, and I. Moroz, "Data assimilation using Bayesian filters and B-spline geological models," in Journal of Physics: Conference Series, vol. 290, no. 1. IOP Publishing, 2011, p. 12004.

[23] J. Mandel, Efficient implementation of the ensemble Kalman filter. University of Colorado at Denver and Health Sciences Center, Center for Computational Mathematics, 2006.

[24] E. J. Candès, J. Romberg, and T. Tao, "Robust uncertainty principles: Exact signal reconstruction from highly incomplete frequency information," Information Theory, IEEE Transactions on, vol. 52, no. 2, pp. 489-509, 2006.

[25] D. L. Donoho, "Compressed sensing," Information Theory, IEEE Transactions on, vol. 52, no. 4, pp. 1289-1306, Apr. 2006.

[26] S. S. Chen, D. L. Donoho, and M. A. Saunders, "Atomic decomposition by basis pursuit," SIAM journal on scientific computing, vol. 20, no. 1, pp. 33-61, 1998.

[27] E. J. Candes, Y. C. Eldar, D. Needell, and P. Randall, "Compressed sensing with coherent and redundant dictionaries," Applied and Computational Harmonic Analysis, vol. 31, no. 1, pp. 59-73, 2011. 
[28] Y. Zhao, A. Reynolds, and G. Li, "Generating facies maps by assimilating production data and seismic data with the ensemble Kalman filter," in SPE/DOE Symposium on Improved Oil Recovery, 2008.

[29] B. Jafarpour, "Wavelet reconstruction of geologic facies from nonlinear dynamic flow measurements," Geoscience and Remote Sensing, IEEE Transactions on, vol. 49, no. 5, pp. 1520-1535, 2011.

[30] M. M. Khaninezhad, B. Jafarpour, and L. Li, "Sparse geologic dictionaries for subsurface flow model calibration: Part II. Robustness to uncertainty," Advances in Water Resources, vol. 39, pp. 106-121, 2012.

[31] I. Hoteit and A. Köhl, "Efficiency of reduced-order, time-dependent adjoint data assimilation approaches," Journal of oceanography, vol. 62, no. 4, pp. 539-550, 2006.

[32] M. U. Altaf, M. Ambrozic, M. F. McCabe, and I. Hoteit, "A study of reduced-order 4DVAR with a finite element shallow water model," International Journal for Numerical Methods in Fluids, 2015.

[33] J. A. Tropp and A. C. Gilbert, "Signal recovery from random measurements via orthogonal matching pursuit," Information Theory, IEEE Transactions on, vol. 53, no. 12, pp. 4655-4666, 2007.

[34] D. L. Donoho, Y. Tsaig, I. Drori, and J.-L. Starck, "Sparse solution of underdetermined systems of linear equations by stagewise orthogonal matching pursuit," Information Theory, IEEE Transactions on, vol. 58, no. 2, pp. 1094-1121, 2012.

[35] K. Lie, S. Krogstad, I. Ligaarden, and Others, "Open-source MATLAB implementation of consistent discretisations on complex grids," Computational Geosciences, vol. 16, no. 2, pp. 297-322, 2012.

[36] Z. Chen, Reservoir Simulation: Mathematical Techniques in Oil Recovery, ser. CBMS-NSF regional conference series in applied mathematics. Society for Industrial and Applied Mathematics (SIAM), 2007.

[37] O. Leeuwenburgh, "EnKF Module for MRST - ISAPP," 2013. [Online]. Available: http://www.isapp2.com/data-sharepoint/enkf-module-formrst 
[38] G. Evensen, "Sequential data assimilation with a nonlinear quasigeostrophic model using Monte Carlo methods to forecast error statistics," Journal of Geophysical Research: Oceans (1978-2012), vol. 99, no. C5, pp. $10143-10162,1994$.

[39] O. Leeuwenburgh, J. Brouwer, and M. Trani, "Ensemble-based conditioning of reservoir models to seismic data," Computational Geosciences, vol. 15, no. 2, pp. 359-378, 2011.

[40] K. Katterbauer and I. Hoteit, "Improving Reservoir History Matching of EM Heated Heavy Oil Reservoirs via Cross-well Seismic Tomography," in SPE Heavy Oil Conference-Canada. Society of Petroleum Engineers, 2014.

[41] K. Katterbauer, S. Arango, S. Sun, and I. Hoteit, "Multidata reservoir history matching for enhanced reservoir forecasting and uncertainty quantification," Journal of Petroleum Science and Engineering, vol. 128, no. 0, pp. 160-176, 2015. [Online]. Available: http://www.sciencedirect.com/science/article/pii/S0920410515000686

[42] X. Luo and I. Hoteit, "Ensemble Kalman filtering with residual nudging," Tellus A, vol. 64, p. 17130, 2012.

[43] I. Hoteit, D.-T. Pham, M. E. Gharamti, and X. Luo, "Mitigating Observation Perturbation Sampling Errors in the Stochastic EnKF," Monthly Weather Review, no. 2015, 2015.

[44] E. J. Candes, J. K. Romberg, and T. Tao, "Stable signal recovery from incomplete and inaccurate measurements," Communications on pure and applied mathematics, vol. 59, no. 8, pp. 1207-1223, 2006.

[45] M. Aharon, M. Elad, and A. Bruckstein, "K-SVD: An Algorithm for Designing Overcomplete Dictionaries for Sparse Representation," IEEE Transactions on Signal Processing, vol. 54, no. 11, pp. 4311-4322, 2006.

[46] T. T. Cai and L. Wang, "Orthogonal matching pursuit for sparse signal recovery with noise," Information Theory, IEEE Transactions on, vol. 57, no. 7, pp. 4680-4688, 2011.

[47] N. Ahmed, T. Natarajan, and K. R. Rao, "Discrete cosine transform," Computers, IEEE Transactions on, vol. 100, no. 1, pp. 90-93, 1974. 
[48] S. G. Mallat, "A theory for multiresolution signal decomposition: the wavelet representation," Pattern Analysis and Machine Intelligence, IEEE Transactions on, vol. 11, no. 7, pp. 674-693, 1989.

[49] R. Rubinstein, A. M. Bruckstein, and M. Elad, "Dictionaries for Sparse Representation Modeling," Proceedings of the IEEE, vol. 98, no. 6, pp. 1045-1057, Jun. 2010.

[50] M. Khodabakhshi, O. P. Corporation, B. Jafarpour, and M. J. King, "Field Applications of a Multi-Scale Multi-Physics History Matching," no. 1997, 2015.

[51] S. Strebelle, "Conditional simulation of complex geological structures using multiple-point statistics," Mathematical Geology, vol. 34, no. 1, pp. 1-21, 2002.

[52] Z. Wang, A. C. Bovik, H. R. Sheikh, and E. P. Simoncelli, "Image quality assessment: from error visibility to structural similarity," Image Processing, IEEE Transactions on, vol. 13, no. 4, pp. 600-612, 2004.

[53] A. Bouzerdoum, "Image quality assessment using a neural network approach," in Signal Processing and Information Technology, Proceedings of the Fourth IEEE International Symposium on, no. Dec, Dec. 2004, pp. 330-333.

[54] D. W. Vasco, A. Ferretti, and F. Novali, "Reservoir monitoring and characterization using satellite geodetic data Interferometric synthetic aperture radar observations from the Krechba field, Algeria," Geophysics, vol. 73, no. 6, pp. WA113 - WA122, 2008.

[55] K. Katterbauer, I. Hoteit, S. Sun, and Others, "Data Assimilation of InSAR Measurements for Large Scale Reservoirs," in SPE Saudi Arabia Section Technical Symposium and Exhibition. Society of Petroleum Engineers, 2014.

[56] A. Tamburini, M. Minini, A. Higgs, G. Falorni, and S. Cespa, "TimeLapse Monitoring with Satellite Data for Reservoir Management," in GEO 2012, 2012. 


\section{List of Tables}

1 Values of the standard deviation of observational errors used in the experimental results shown in Figure 11 . . . . . . . . 38 


\begin{tabular}{|c|c|c|c|}
\hline & Flux $\left(m^{3} /\right.$ day $)$ & BHP $($ Psi) & WCT $(\%)$ \\
\hline \hline Case 1 & 25 & 290 & 7 \\
\hline Case 2 & 50 & 580 & 10 \\
\hline Case 3 & 100 & 1160 & 15 \\
\hline
\end{tabular}

Table 1: Values of the standard deviation of observational errors used in the experimental results shown in Figure 11 


\section{List of Figures}

1 (a): Reference permeability field composed of high permeability areas plotted in red indicating flow channels and low permeability areas plotted in blue indicating surrounding rock formations. (b)-(d): Injector-producer patterns showing three different distributions of the injector wells (black circles) and producer wells (white circles) on top of the reference permeability field. . . . . . . . . . . . . . . . . . 46

2 The training image in panel (a) is used to generate multiple realizations of subsurface geological structures (examples of these are shown in panel (b)) using the SNESIM algorithm. These realizations are later used as input to the K-SVD algorithm to generate a dictionary of linearly independent basis elements as outlined in panel (c). A weighted sparse linear combination of these basis elements, using the OMP algorithm, can be used to re-generate any of the original realizations or to represent them in the sparse dictionary domain, as schematized in panel $(\mathrm{d}) . \ldots \ldots \ldots$. . . . . . . . 47 
3 Flowchart representation of the Ensemble Kalman Filter (EnKF) based history matching framework. A state vector (panel (a)) consisting of the reservoir parameters, such as permeability, porosity, saturation, etc., is fed into the simulator (panel (b)). The reservoir simulator is then used to forecast the future state of the reservoir, which is then sent to the EnKF module (schematized in panel (c)) where it is updated by incoming new observations. For the next filtering cycle, these updated reservoir parameters are sent back to the simulator to perform the next forecast and the process continues until the end of the history matching period. . . . . . . . . . . . . . . 48

4 Recovered permeability field as it results from the standard EnKF (left column), SGSD-EnKF (middle column), and DEUEnKF (right column) over the 5-years history matching period. Rows top-to-bottom represent the recovered fields after 1, 2, 3, 4 and 5 years of history matching, respectively. All values and scales are in units of millidarcy $(\mathrm{mD})$. The filters are implemented with an ensemble of 80 members, using the injector-producer pattern A shown in Figure 1, and 10\% basis elements from the K-SVD dictionary for the SGSD-EnKF. . . 49 
5 Estimation quality of the recovered permeability field, in terms of the SSIM and CEE, over the 5-years history matching period for the experiment shown in Figure 4. Panel (a) compares the performances of the standard EnKF, SGSD-EnKF, and DEU-EnKF based on the SSIM index (as explained in Section 5.1), where a high value of SSIM indicates that the estimated field resembles more closely to the reference permeability field shown in Figure 1. Panel (b) performs a similar comparison of the estimated fields in terms of the CEE (defined in Section 5.1), where a low CEE value indicates more accurate estimates. 50

6 Sensitivity analysis of the quality of the recovered permeability field as it results from the SGSD-EnKF, in terms of SSIM, with respect to different sparsity rates at the end of the 5-years history matching period for the experiment shown in Figure 4. The results indicate that the best performance is achieved using $10 \%$ basis elements from the dictionary. . . . . . . . . 51 
7 Sensitivity analysis of the quality of the recovered permeability field as it results from the standard EnKF, SGSD-EnKF, and DEU-EnKF, in terms of CEE, with respect to different threshold levels at the end of the 5-years history matching period for the experiment shown in Figure 4. The formulation of the CEE metric is dependent on a threshold level, as explained in Section 5.1. The results suggest that the proposed SGSDEnKF and DEU-EnKF algorithms outperform the standard EnKF for any chosen value of the threshold above 1500 millidarcy. . . . . . . . . . . . . . . . 52

8 Recovered permeability field as it results from the standard EnKF (left panel), DCT representation based EnKF (middle panel), and K-SVD geologic dictionaries based SGSD-EnKF (right panel). All values and scales are in units of millidarcy. This figure highlights the advantage of the K-SVD dictionary based SGSD-EnKF in recovering the channel structures compared to the standard EnKF and an EnKF using a DCT dictionary. The filters were implemented with an ensemble of 80 members, using injector-producer pattern A of Figure 1, and $10 \%$ basis elements for both DCT and K-SVD dictionaries. . 53 
9 Recovered permeability field as it results from the standard EnKF (left column), SGSD-EnKF (middle column), and DEUEnKF (right column) at the end of the 5-years history matching period using different ensemble sizes. Rows top-to-bottom represent the recovered fields using 40, 80, 120, 160 and 200 members, respectively. All values and scales are in units of millidarcy $(\mathrm{mD})$. The experiment was performed using the injector-producer pattern A of Figure 1, and using 10\% basis elements from the K-SVD dictionary for the SGSD-EnKF.

10 Sensitivity analysis of the quality of the recovered permeability field as it results from the standard EnKF, SGSD-EnKF, and DEU-EnKF, in terms of CEE, with respect to different ensemble sizes at the end of the 5-years history matching period for the experiment shown in Figure 9. The results indicate that the proposed SGSD-EnKF and DEU-EnKF have lower CEE values than the standard EnKF for any given number of ensemble members, suggesting better recovery of the channels structures. . . . . . . . . . . . . . . 55 
11 Recovered permeability field as it results from the standard EnKF (left column), SGSD-EnKF (middle column) and DEUEnKF (right column) at the end of 5-years history matching period using different values of observational error standard deviation. Rows top-to-bottom represent the recovered fields for Cases 1, 2, and 3 as described in Table 1, respectively. All values and scales are in units of millidarcy $(\mathrm{mD})$. The filters were implemented with an ensemble of 80 members, using the injector-producer pattern A of Figure 1, and $10 \%$ basis elements from the K-SVD dictionary for the SGSD-EnKF. 56

12 Sensitivity analysis of the quality of the recovered permeability field as it results from the standard EnKF, SGSD-EnKF, and DEU-EnKF, in terms of CEE, with respect to increasing observational error standard deviation at the end of the 5-years history matching period for the experiment shown in Figure 11. The results indicate that the proposed SGSD-EnKF and DEU-EnKF filters achieve lower CEE values than the standard EnKF even when the standard deviation of observational error increases, and hence are more robust in terms of channels recovery. . . . . . . . . . . . . . . . . 57 
13 Recovered permeability field as it results from the standard EnKF (left column), SGSD-EnKF (middle column), and DEUEnKF (right column) at the end of the 5-years history matching period using different injector-producer patterns. Rows top-to-bottom represent the field recovered using the injectorproducer patterns B and C, as shown in Figure 1, respectively. All values and scales are in units of millidarcy $(\mathrm{mD})$. The filters were implemented with an ensemble size of 80 members and using $10 \%$ basis elements from the K-SVD dictionary for the SGSD-EnKF. . . . . . . . . . . . . . . . . . 58

14 Sensitivity analysis of the quality of the recovered permeability field as it results from the standard EnKF, SGSD-EnKF, and DEU-EnKF, in terms of CEE, with respect to different injector-producer patterns at the end of the 5-years history matching period for the experiment shown in Figure 13. The results indicate that the proposed DEU-EnKF significantly outperforms the standard EnKF in recovering the channels structures using any of the tested injector-producer patterns. The SGSD-EnKF exhibits significant advantage over the standard EnKF when less number of observation points (wells) are available. . . . . . . . . . . . . . . . 59 
(a) Reference Permeability Field

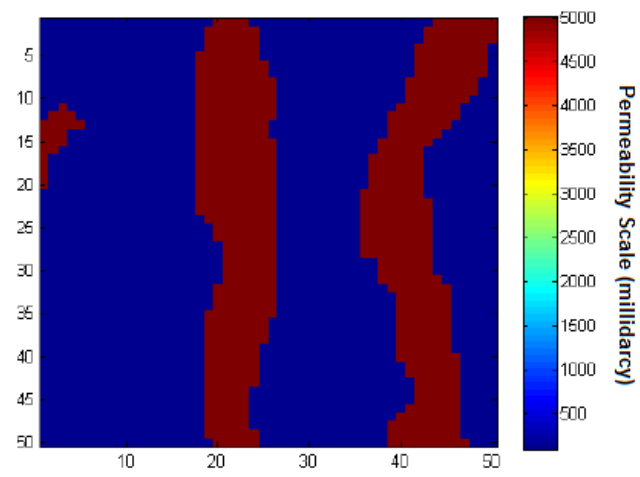

(c) Injector-Producer Pattern B

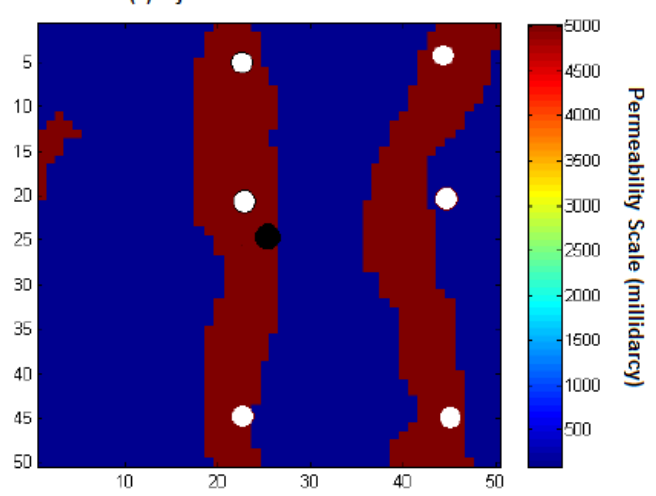

(b) Injector-Producer Pattern A

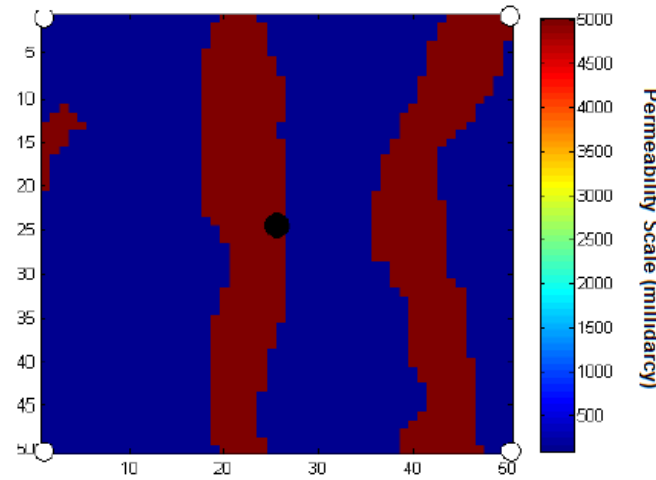

(d) Injector-Producer Pattern C

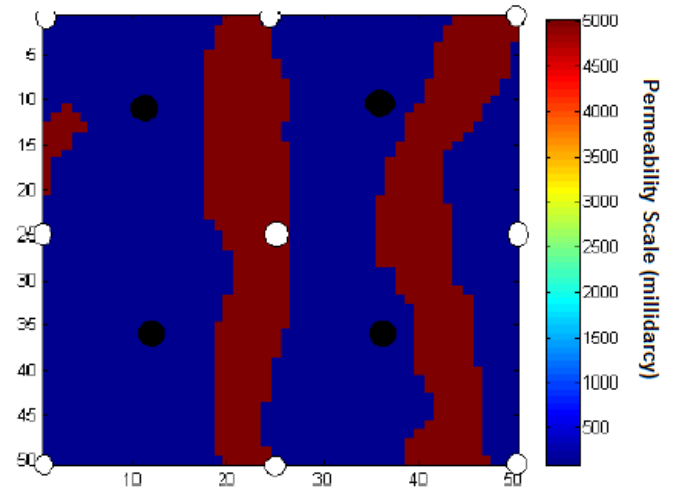

Figure 1: (a): Reference permeability field composed of high permeability areas plotted in red indicating flow channels and low permeability areas plotted in blue indicating surrounding rock formations. (b)-(d): Injector-producer patterns showing three different distributions of the injector wells (black circles) and producer wells (white circles) on top of the reference permeability field. 
(a) Input to SNESIM Algorithm: Training Image

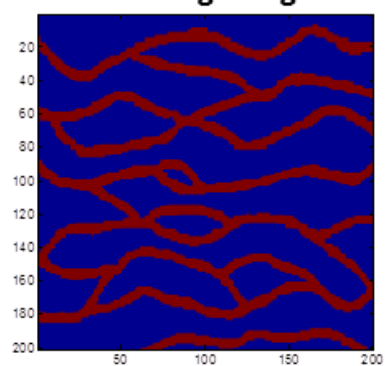

\section{SNESIM}
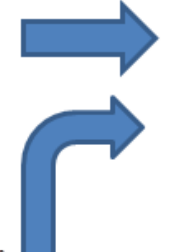

d) Weights for Sparse Domain Representation of Different Geological Structures

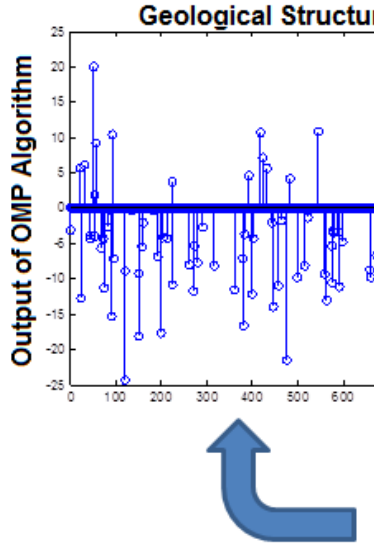

(b) Output of SNESIM Algorithm: Multiple Realizations of Complex Geological Structures
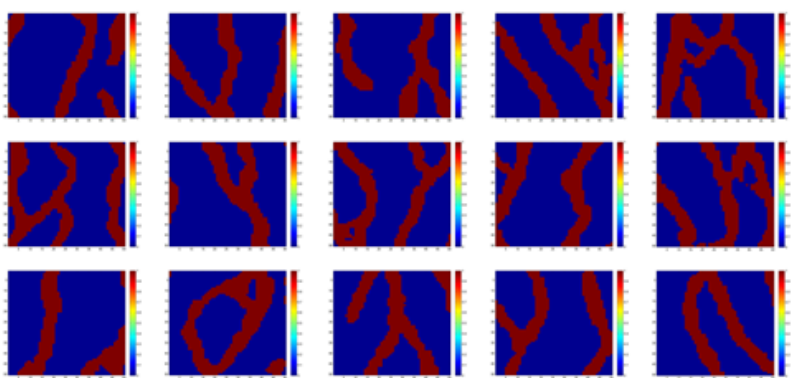

$$
\begin{aligned}
& \text { 政 } \min _{\mathbf{X}, \boldsymbol{\Psi}}\left\{\|\mathbf{Y}-\boldsymbol{\Psi} \mathbf{X}\|_{2}^{2}\right\} \\
& \text { s.t. }\left\|\mathbf{x}_{i}\right\|_{0} \leq \mathbf{T}_{0} \forall i=1, \ldots, N_{t}
\end{aligned}
$$
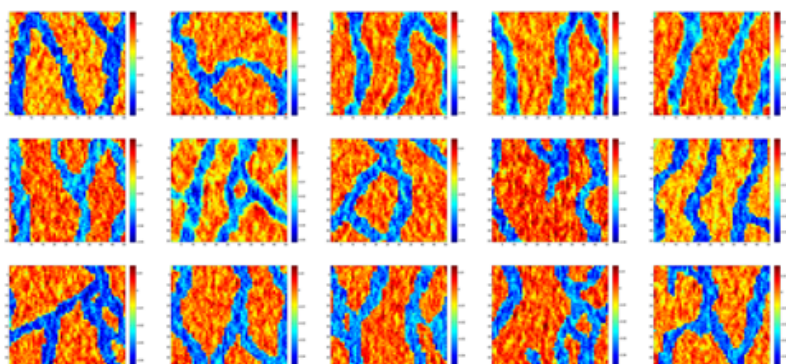

(c) Dictionary

$\min _{\mathbf{x}}\|\mathbf{x}\|_{0}$ subject to: $\left\|\mathbf{y}-\Psi_{\mathbf{x}}\right\|_{2} \leq \eta$,

Orthogonal Matching Pursuit (OMP)

Figure 2: The training image in panel (a) is used to generate multiple realizations of subsurface geological structures (examples of these are shown in panel (b)) using the SNESIM algorithm. These realizations are later used as input to the K-SVD algorithm to generate a dictionary of linearly independent basis elements as outlined in panel (c). A weighted sparse linear combination of these basis elements, using the OMP algorithm, can be used to re-generate any of the original realizations or to represent them in the sparse dictionary domain, as schematized in panel (d). 
(a) Geological Model • Geological Structure

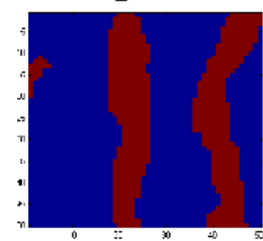

- Number of Wells

- Pressure

- Saturation

- Permeability

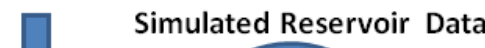

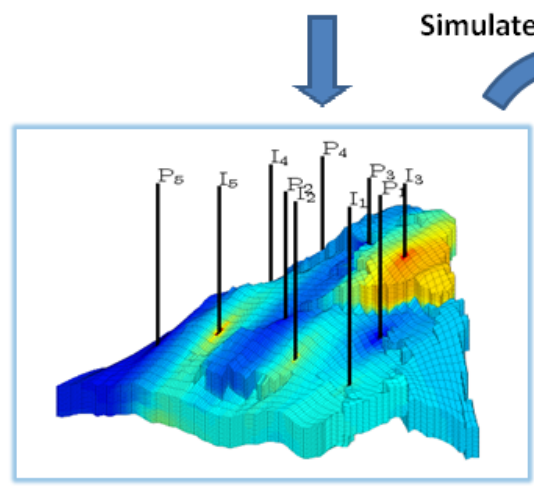

(b) Reservoir Simulator
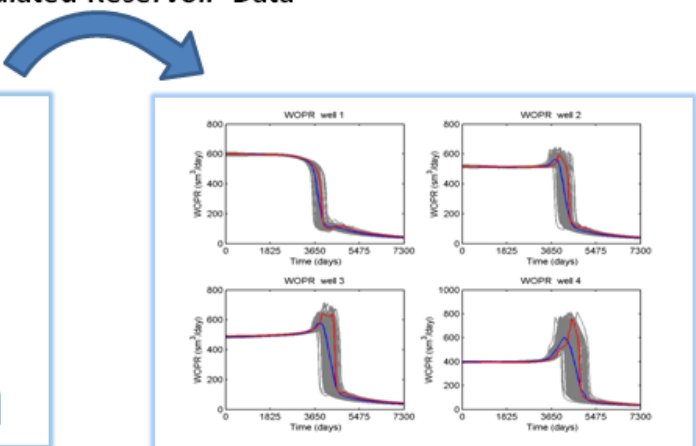

(c) EnKF based History Matching

Calibrated Reservoir Parameters

Figure 3: Flowchart representation of the Ensemble Kalman Filter (EnKF) based history matching framework. A state vector (panel (a)) consisting of the reservoir parameters, such as permeability, porosity, saturation, etc., is fed into the simulator (panel (b)). The reservoir simulator is then used to forecast the future state of the reservoir, which is then sent to the EnKF module (schematized in panel (c)) where it is updated by incoming new observations. For the next filtering cycle, these updated reservoir parameters are sent back to the simulator to perform the next forecast and the process continues until the end of the history matching period. 

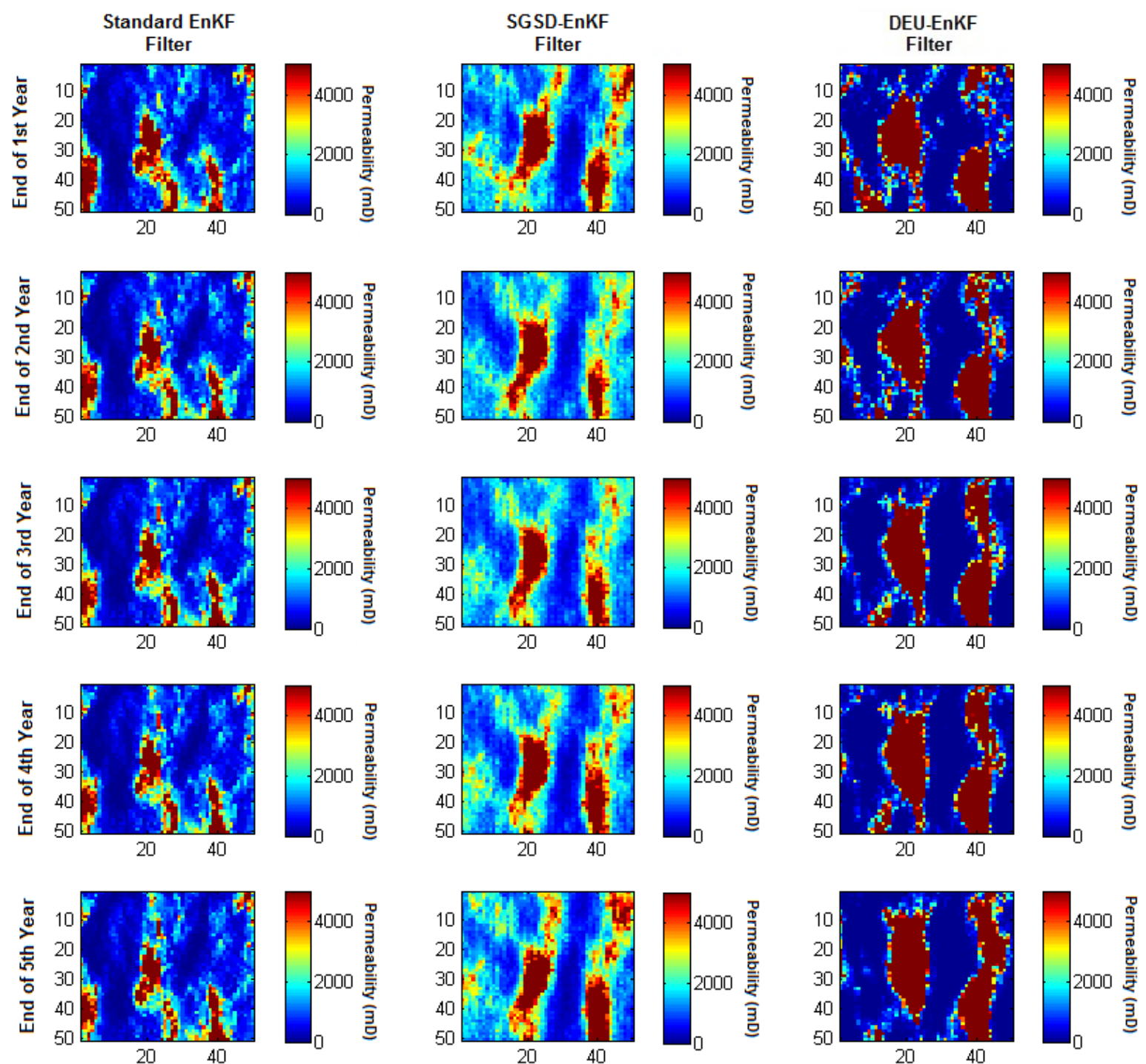

Figure 4: Recovered permeability field as it results from the standard EnKF (left column), SGSD-EnKF (middle column), and DEU-EnKF (right column) over the 5-years history matching period. Rows top-to-bottom represent the recovered fields after 1, 2, 3, 4 and 5 years of history matching, respectively. All values and scales are in units of millidarcy $(\mathrm{mD})$. The filters are implemented with an ensemble of 80 members, using the injectorproducer pattern A shown in Figure 1, and 10\% basis elements from the K-SVD dictionary for the SGSD-EnKF. 


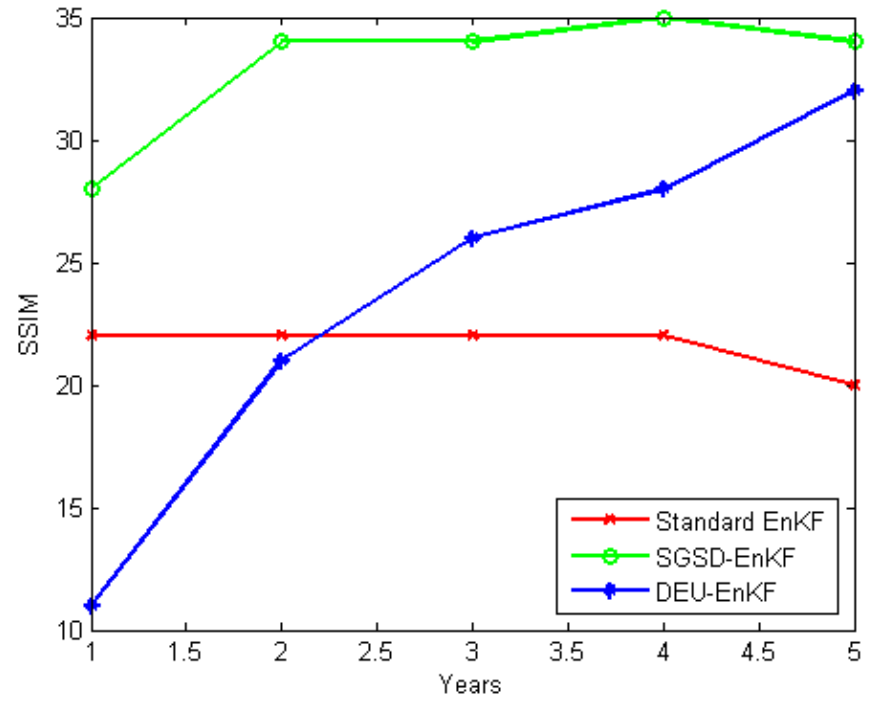

(a)

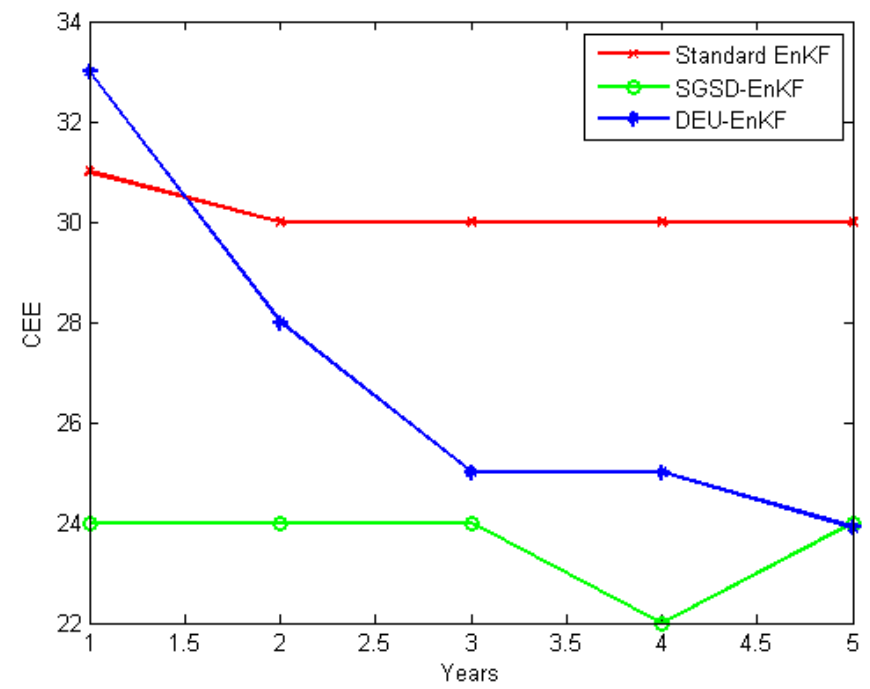

(b)

Figure 5: Estimation quality of the recovered permeability field, in terms of the SSIM and $\mathrm{CEE}$, over the 5-years history matching period for the experiment shown in Figure 4. Panel (a) compares the performances of the standard EnKF, SGSD-EnKF, and DEUEnKF based on the SSIM index (as explained in Section 5.1), where a high value of SSIM indicates that the estimated field resembles more closely to the reference permeability field shown in Figure 1. Panel (b) performs a similar comparison of the estimated fields in terms of the CEE (defined in Section 5.1), where a low CEE value indicates more accurate estimates. 


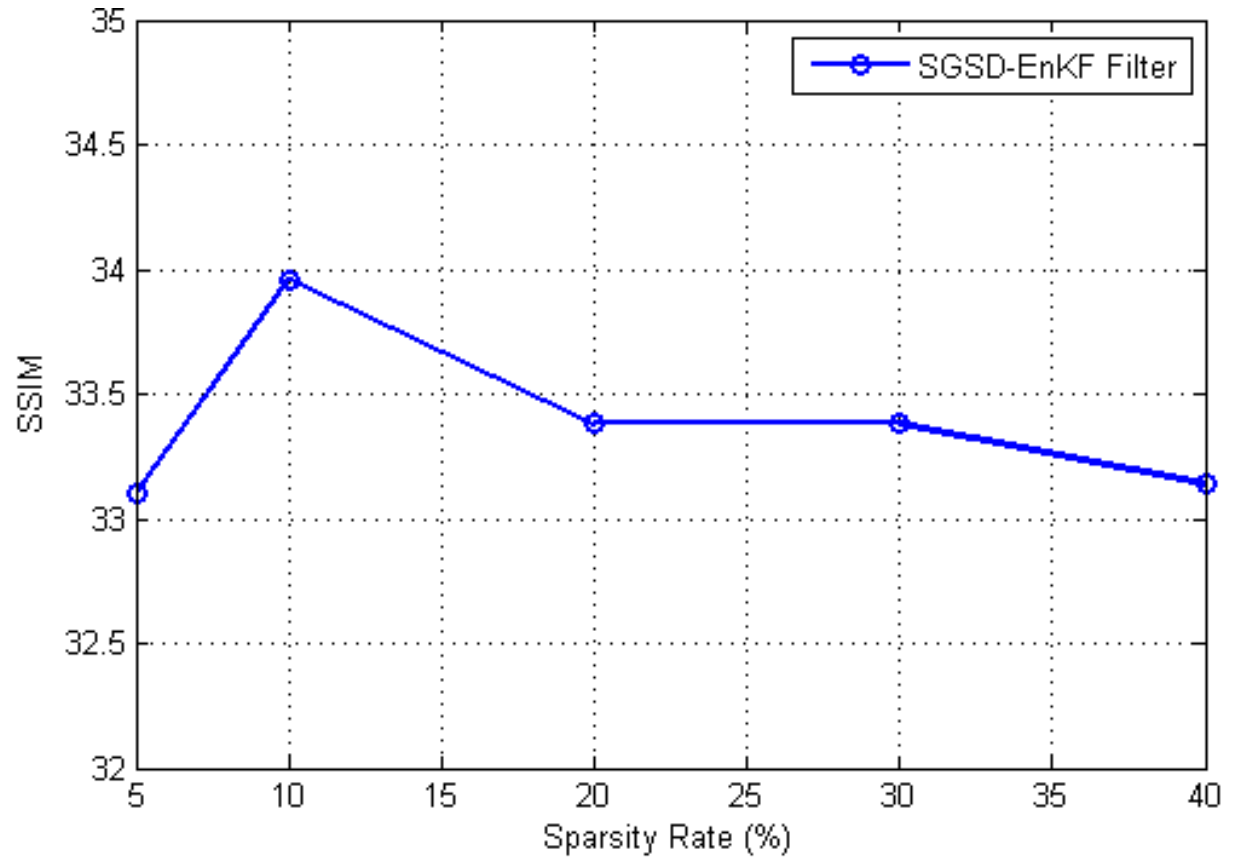

Figure 6: Sensitivity analysis of the quality of the recovered permeability field as it results from the SGSD-EnKF, in terms of SSIM, with respect to different sparsity rates at the end of the 5-years history matching period for the experiment shown in Figure 4. The results indicate that the best performance is achieved using $10 \%$ basis elements from the dictionary. 


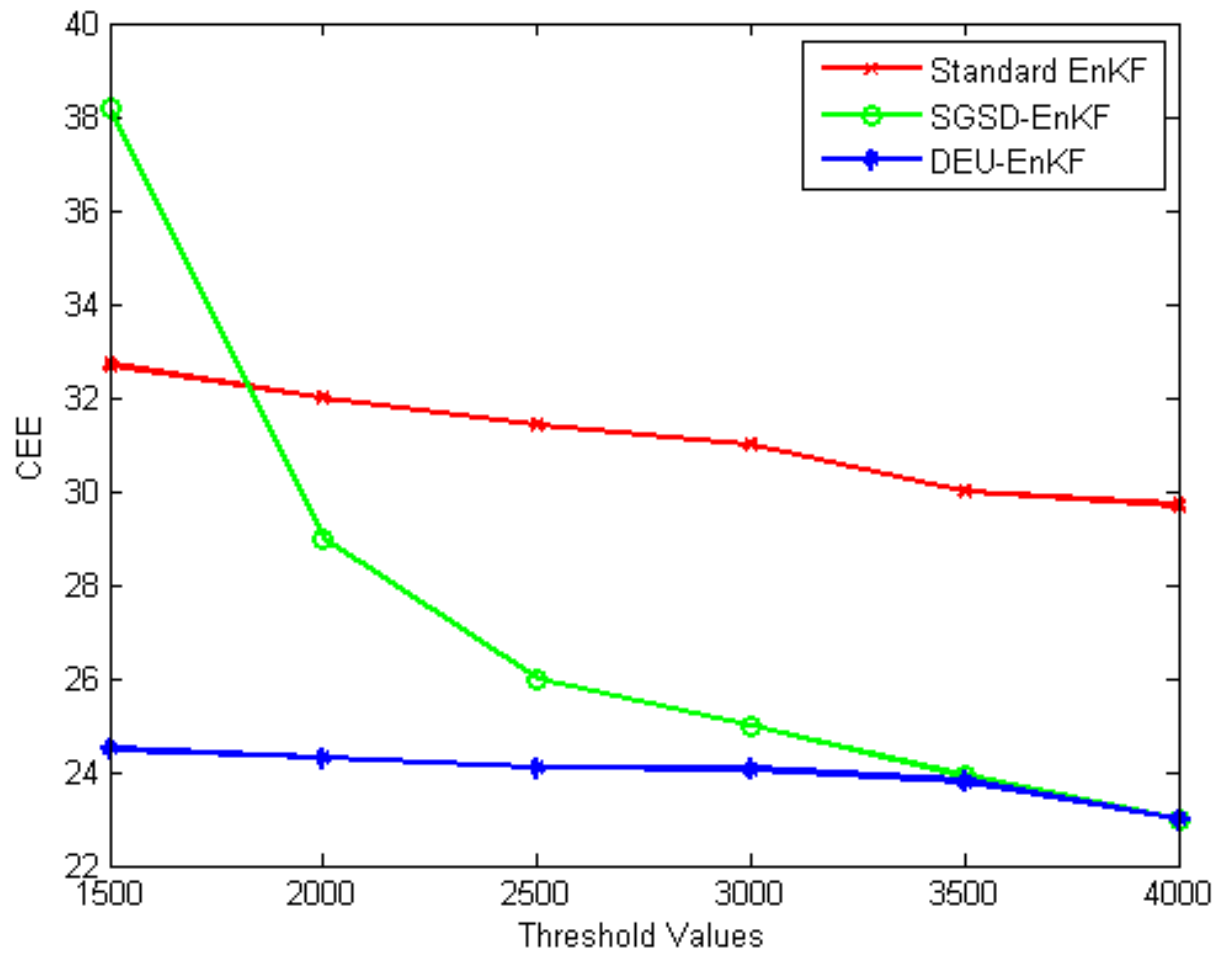

Figure 7: Sensitivity analysis of the quality of the recovered permeability field as it results from the standard EnKF, SGSD-EnKF, and DEU-EnKF, in terms of CEE, with respect to different threshold levels at the end of the 5-years history matching period for the experiment shown in Figure 4. The formulation of the CEE metric is dependent on a threshold level, as explained in Section 5.1. The results suggest that the proposed SGSDEnKF and DEU-EnKF algorithms outperform the standard EnKF for any chosen value of the threshold above 1500 millidarcy. 

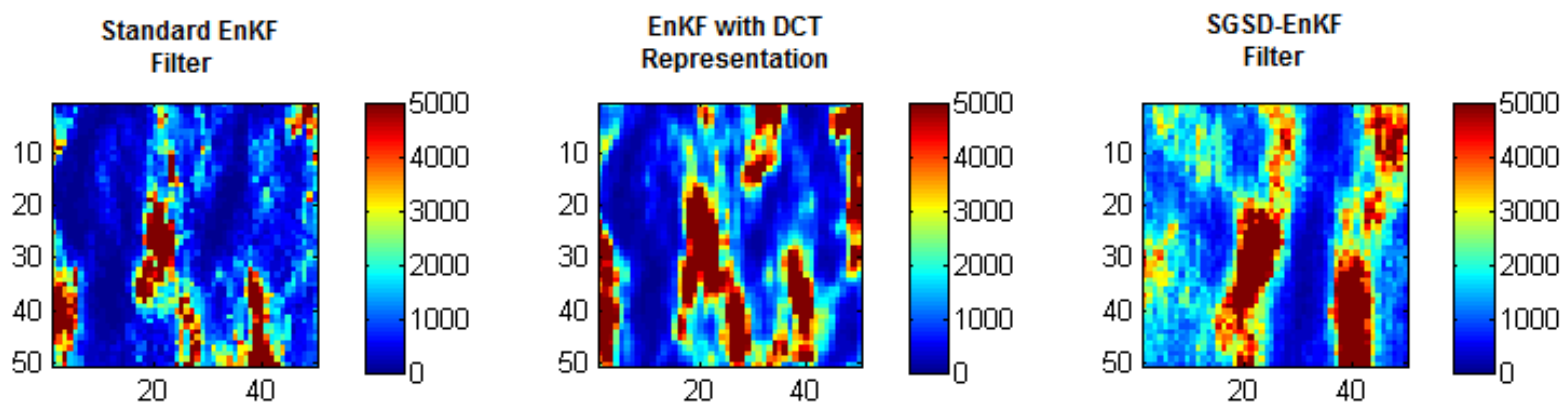

Figure 8: Recovered permeability field as it results from the standard EnKF (left panel), DCT representation based EnKF (middle panel), and K-SVD geologic dictionaries based SGSD-EnKF (right panel). All values and scales are in units of millidarcy. This figure highlights the advantage of the K-SVD dictionary based SGSD-EnKF in recovering the channel structures compared to the standard EnKF and an EnKF using a DCT dictionary. The filters were implemented with an ensemble of 80 members, using injector-producer pattern A of Figure 1, and 10\% basis elements for both DCT and K-SVD dictionaries. 

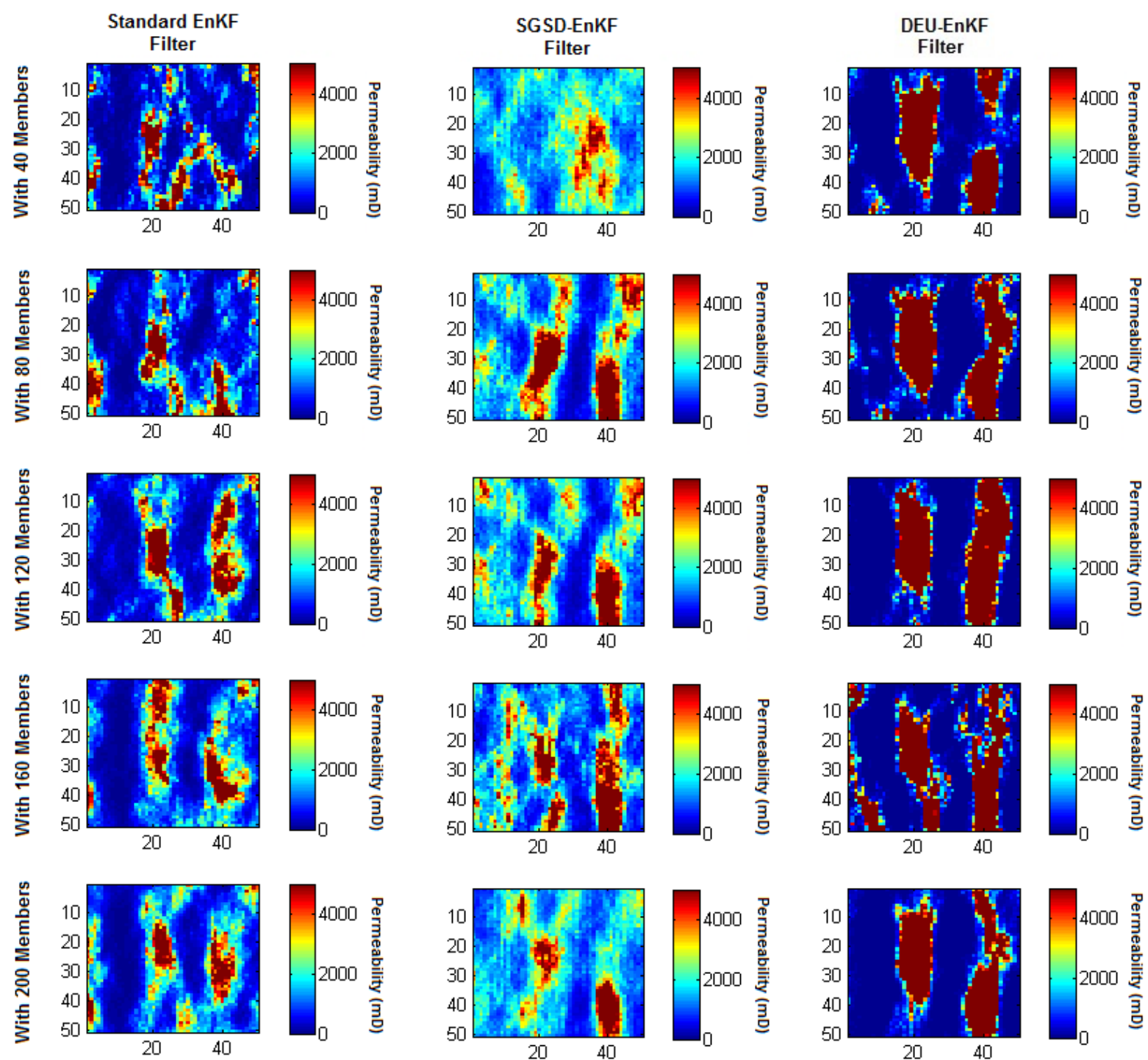

Figure 9: Recovered permeability field as it results from the standard EnKF (left column), SGSD-EnKF (middle column), and DEU-EnKF (right column) at the end of the 5-years history matching period using different ensemble sizes. Rows top-to-bottom represent the recovered fields using 40, 80, 120, 160 and 200 members, respectively. All values and scales are in units of millidarcy $(\mathrm{mD})$. The experiment was performed using the injector-producer pattern A of Figure 1, and using $10 \%$ basis elements from the K-SVD dictionary for the SGSD-EnKF. 


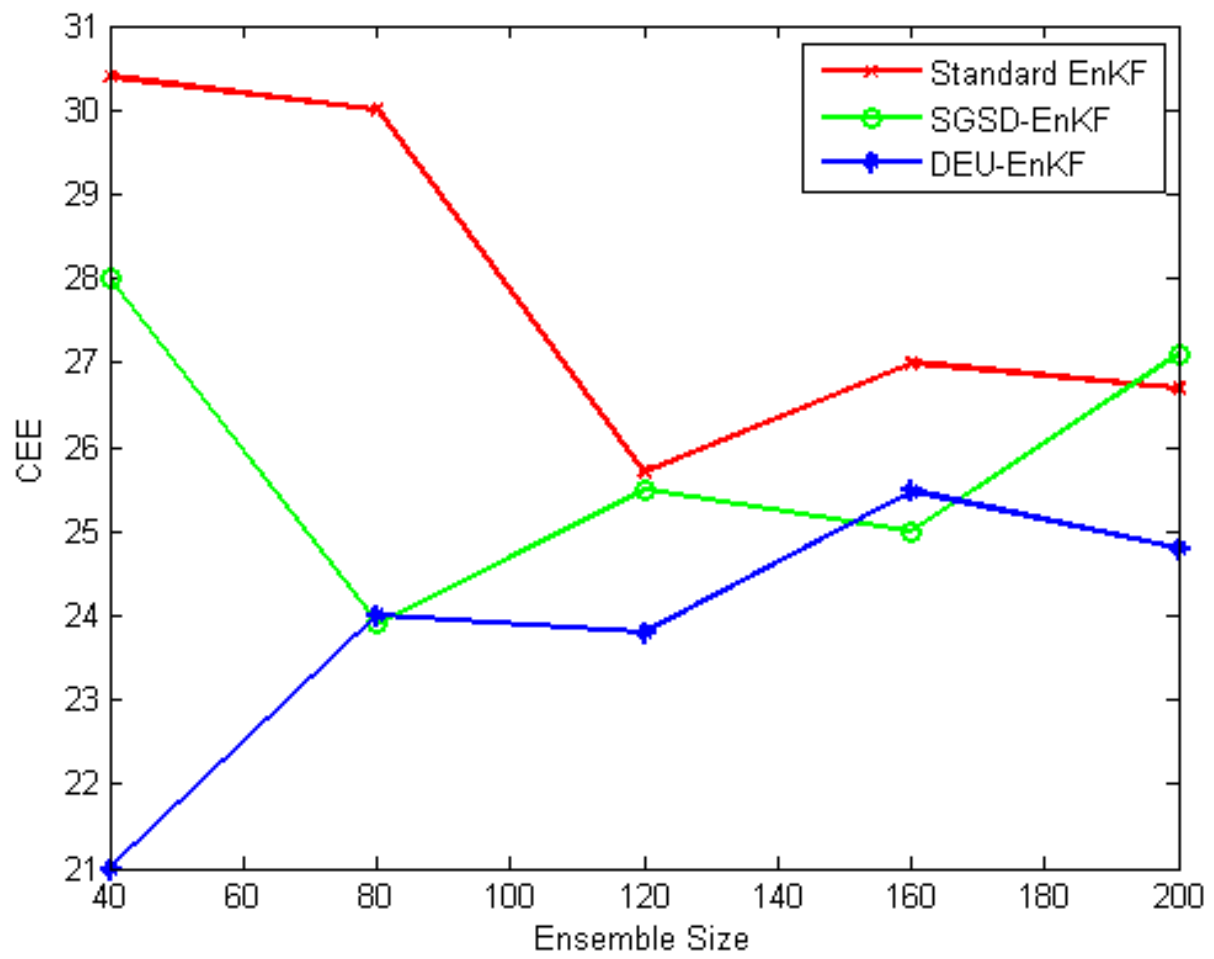

Figure 10: Sensitivity analysis of the quality of the recovered permeability field as it results from the standard EnKF, SGSD-EnKF, and DEU-EnKF, in terms of CEE, with respect to different ensemble sizes at the end of the 5-years history matching period for the experiment shown in Figure 9. The results indicate that the proposed SGSD-EnKF and DEU-EnKF have lower CEE values than the standard EnKF for any given number of ensemble members, suggesting better recovery of the channels structures. 

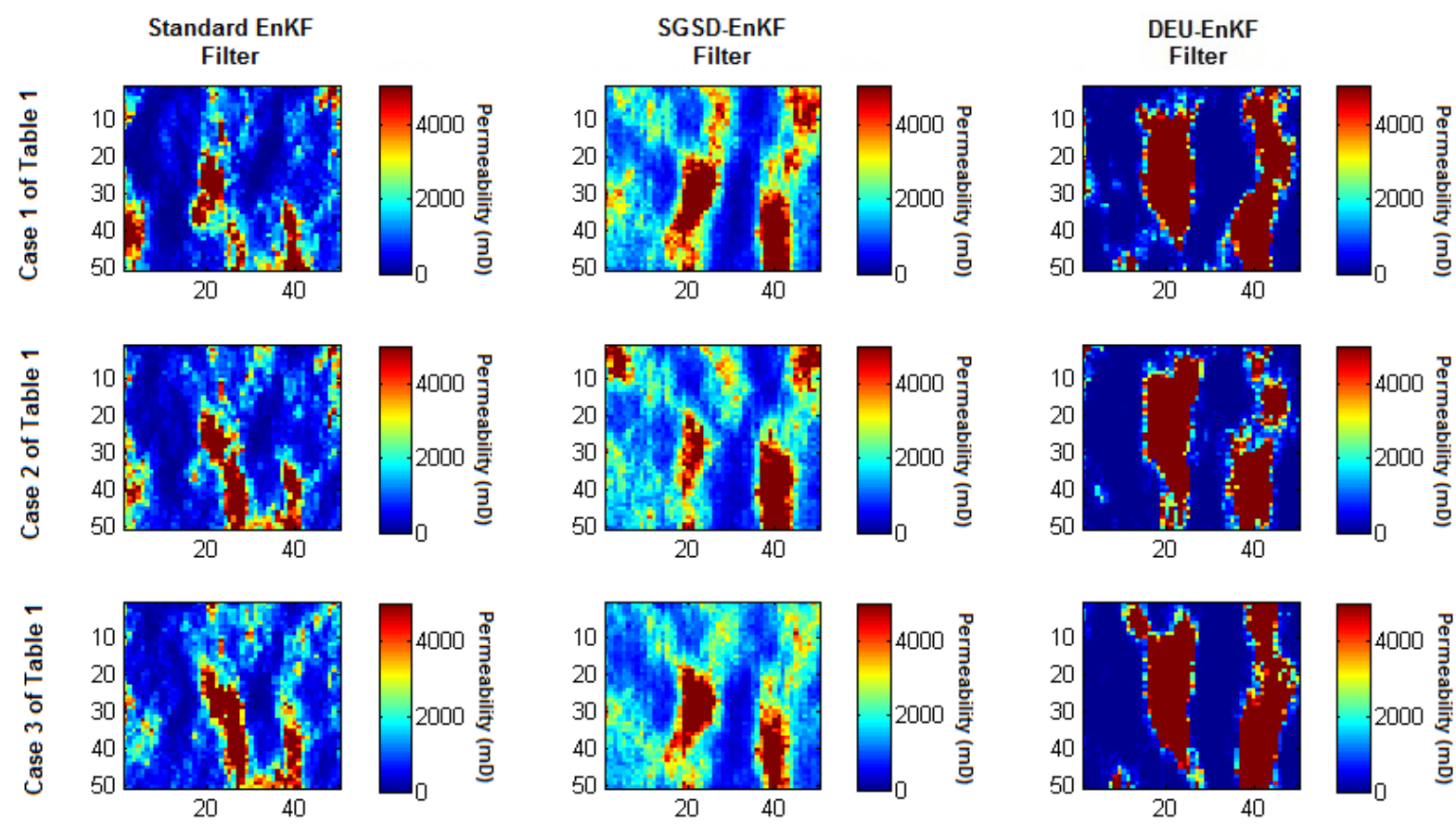

Figure 11: Recovered permeability field as it results from the standard EnKF (left column), SGSD-EnKF (middle column) and DEU-EnKF (right column) at the end of 5-years history matching period using different values of observational error standard deviation. Rows top-to-bottom represent the recovered fields for Cases 1, 2, and 3 as described in Table 1 , respectively. All values and scales are in units of millidarcy $(\mathrm{mD})$. The filters were implemented with an ensemble of 80 members, using the injector-producer pattern A of Figure 1, and 10\% basis elements from the K-SVD dictionary for the SGSD-EnKF. 


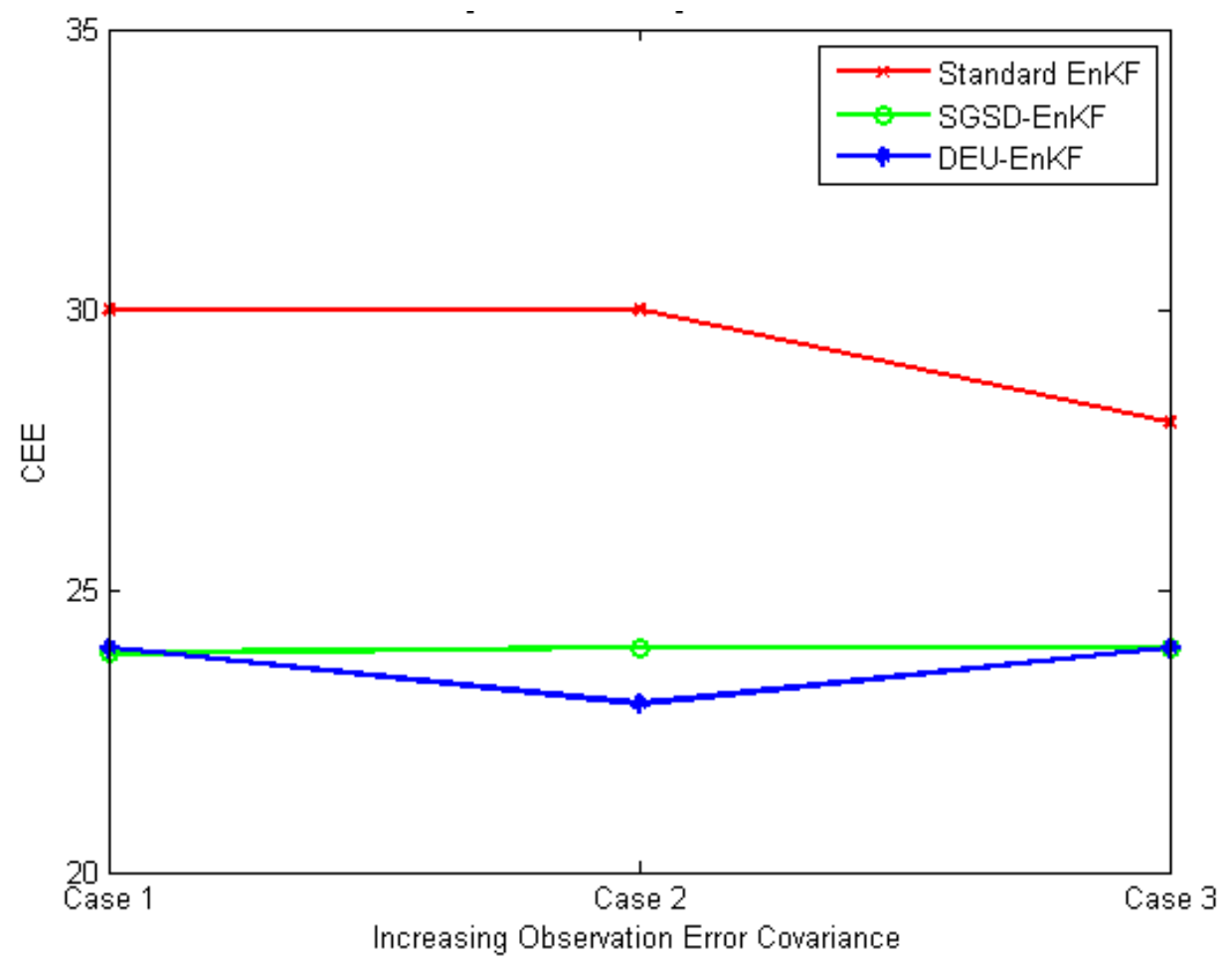

Figure 12: Sensitivity analysis of the quality of the recovered permeability field as it results from the standard EnKF, SGSD-EnKF, and DEU-EnKF, in terms of CEE, with respect to increasing observational error standard deviation at the end of the 5-years history matching period for the experiment shown in Figure 11. The results indicate that the proposed SGSD-EnKF and DEU-EnKF filters achieve lower CEE values than the standard EnKF even when the standard deviation of observational error increases, and hence are more robust in terms of channels recovery. 

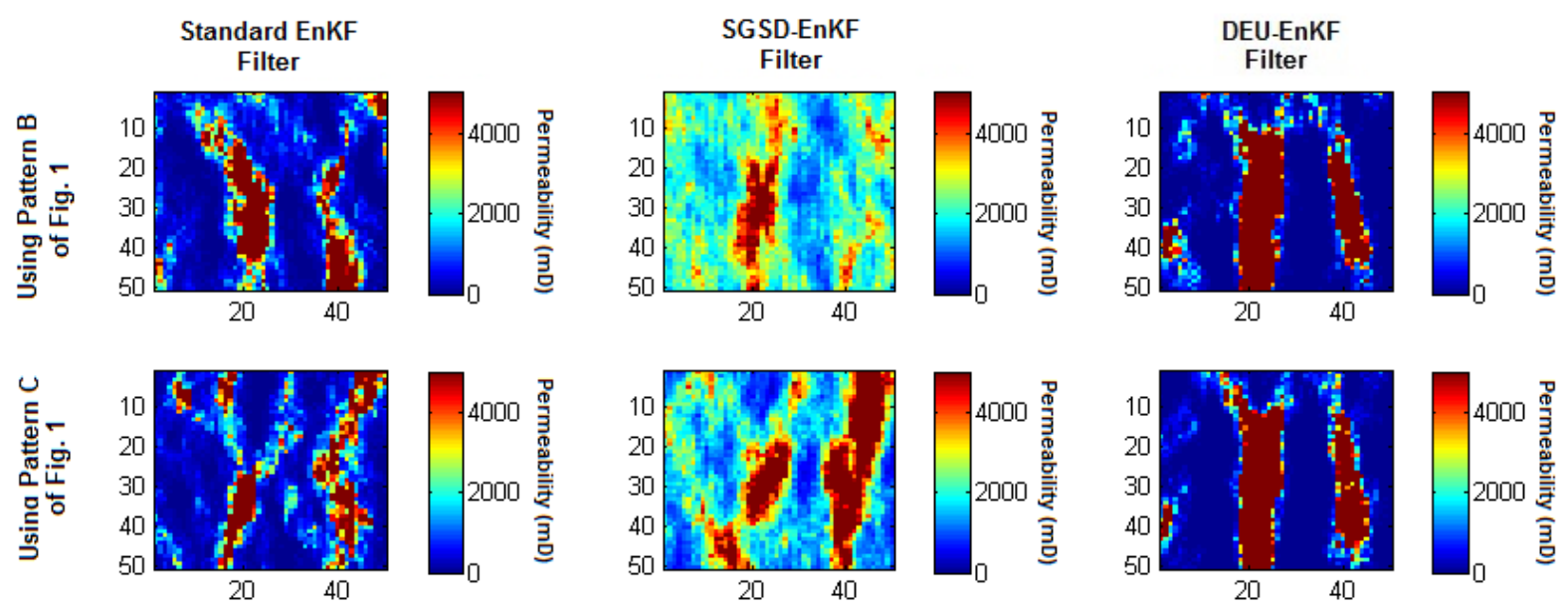

Figure 13: Recovered permeability field as it results from the standard EnKF (left column), SGSD-EnKF (middle column), and DEU-EnKF (right column) at the end of the 5-years history matching period using different injector-producer patterns. Rows top-to-bottom represent the field recovered using the injector-producer patterns $\mathrm{B}$ and $\mathrm{C}$, as shown in Figure 1, respectively. All values and scales are in units of millidarcy $(\mathrm{mD})$. The filters were implemented with an ensemble size of 80 members and using $10 \%$ basis elements from the K-SVD dictionary for the SGSD-EnKF. 


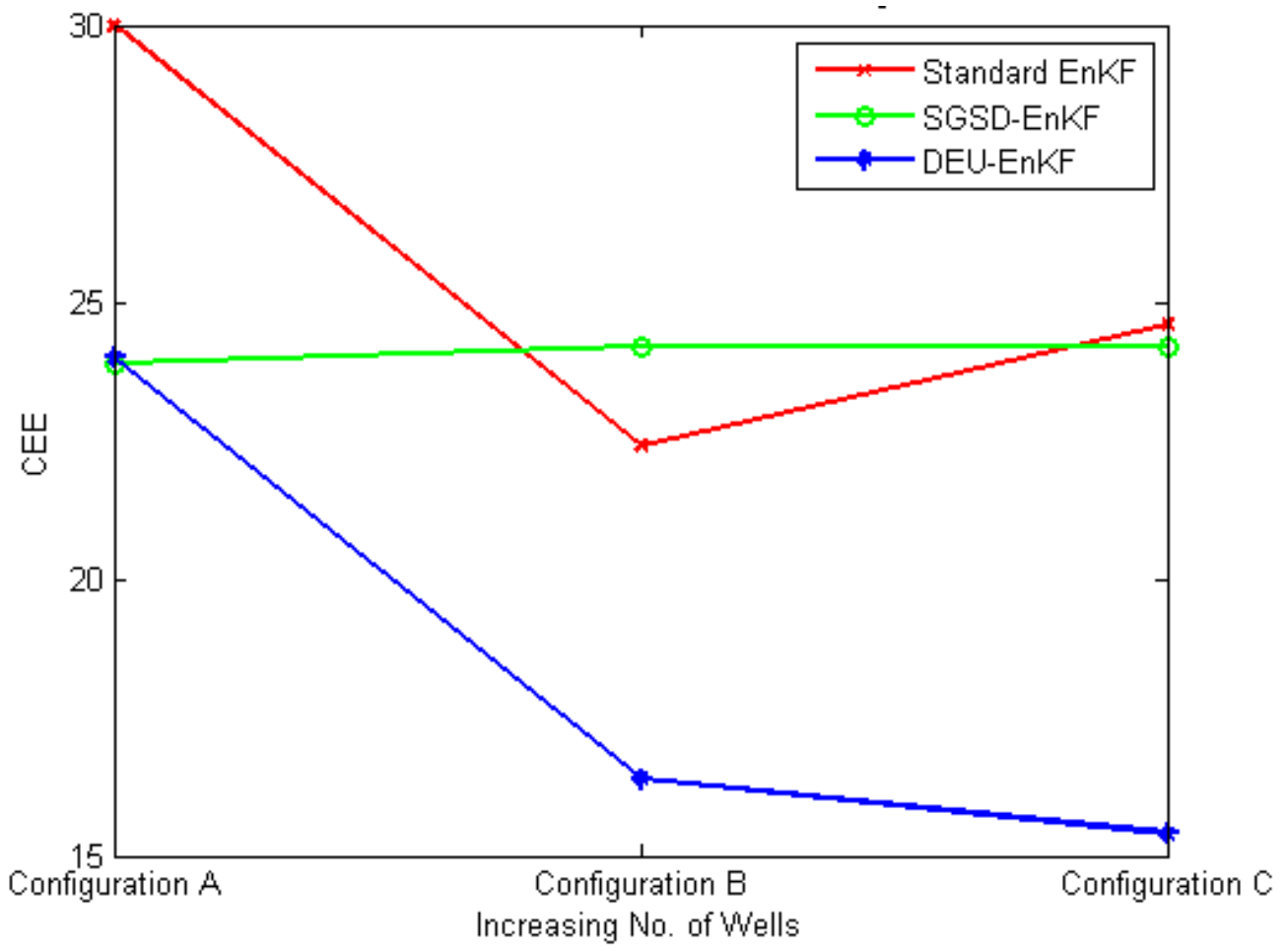

Figure 14: Sensitivity analysis of the quality of the recovered permeability field as it results from the standard EnKF, SGSD-EnKF, and DEU-EnKF, in terms of CEE, with respect to different injector-producer patterns at the end of the 5-years history matching period for the experiment shown in Figure 13. The results indicate that the proposed DEUEnKF significantly outperforms the standard EnKF in recovering the channels structures using any of the tested injector-producer patterns. The SGSD-EnKF exhibits significant advantage over the standard EnKF when less number of observation points (wells) are available. 